\title{
Second-Order PDEs in 3D with Einstein-Weyl Conformal Structure
}

\author{
S. Berjawi, E. V. Ferapontov®, B. S. Kruglikov and V. S. Novikov
}

\begin{abstract}
Einstein-Weyl geometry is a triple $(\mathbb{D}, g, \omega)$ where $\mathbb{D}$ is a symmetric connection, $[g]$ is a conformal structure and $\omega$ is a covector such that $\bullet$ connection $\mathbb{D}$ preserves the conformal class $[g]$, that is, $\mathbb{D} g=$ $\omega g$; • trace-free part of the symmetrised Ricci tensor of $\mathbb{D}$ vanishes. Three-dimensional Einstein-Weyl structures naturally arise on solutions of second-order dispersionless integrable PDEs in 3D. In this context, $[g]$ coincides with the characteristic conformal structure and is therefore uniquely determined by the equation. On the contrary, covector $\omega$ is a somewhat more mysterious object, recovered from the Einstein-Weyl conditions. We demonstrate that, for generic second-order PDEs (for instance, for all equations not of Monge-Ampère type), the covector $\omega$ is also expressible in terms of the equation, thus providing an efficient 'dispersionless integrability test'. The knowledge of $g$ and $\omega$ provides a dispersionless Lax pair by an explicit formula which is apparently new. Some partial classification results of PDEs with Einstein-Weyl characteristic conformal structure are obtained. A rigidity conjecture is proposed according to which for any generic second-order PDE with Einstein-Weyl property, all dependence on the 1 -jet variables can be eliminated via a suitable contact transformation.
\end{abstract}

Mathematics Subject Classification. 35L70, 35Q75, 35Q76, 37K25, 53A40, 53B50, 53C25.

Keywords. Characteristic variety, Einstein-Weyl geometry, Dispersionless integrability, Dispersionless Lax pair, Monge-Ampère property, Contact symmetry. 


\section{Introduction}

We consider second-order partial differential equations (PDEs) in 3D,

$$
F\left(x^{i}, u, u_{i}, u_{i j}\right)=0,
$$

where $u$ is a scalar function of the three independent variables $x^{0}, x^{1}, x^{2}$, and we denote $u_{i}=u_{x^{i}}, u_{i j}=u_{x^{i} x^{j}}$, etc. For every solution of equation (1) the corresponding characteristic variety,

$$
\sum_{i \leq j} \frac{\partial F}{\partial u_{i j}} p_{i} p_{j}=0,
$$

defines a conformal structure $g=g_{i j} d x^{i} d x^{j}$ where the symmetric matrix $\left(g_{i j}\right)_{3 \times 3}$ is inverse to the matrix of the symbol $\left(g^{i j}\right)_{3 \times 3}$, with $g^{i j}=\frac{1+\delta_{i j}}{2} \frac{\partial F}{\partial u_{i j}}$ (no summation). Here and in what follows we assume the nondegeneracy condition $\operatorname{det} g^{i j} \not \equiv 0$, i.e. $[g]$ is well-defined on a generic solution of (1). Equations with nondegenerate characteristic variety will be called nondegenerate.

We will be interested in equations (1) whose characteristic conformal structure $g$ satisfies the Einstein-Weyl property on every solution of (1) (PDEs with EW property for short). Recall that Einstein-Weyl geometry is defined by a triple $(\mathbb{D}, g, \omega)$ where $\mathbb{D}$ is a symmetric connection, $g$ is a conformal structure and $\omega$ is a covector such that [6]:

(a) Connection $\mathbb{D}$ preserves the conformal class $[g]: \mathbb{D} g=\omega g$;

(b) Trace-free part of the symmetrised Ricci tensor of $\mathbb{D}$ vanishes.

In coordinates, this gives

$$
\mathbb{D}_{k} g_{i j}=\omega_{k} g_{i j}, R_{(i j)}=\Lambda g_{i j},
$$

where $\omega=\omega_{k} d x^{k}$ is a covector, $\mathbb{D}_{k}$ denotes covariant derivative, $R_{(i j)}$ is the symmetrised Ricci tensor of $\mathbb{D}$, and $\Lambda$ is some function. In fact one needs to specify $g$ and $\omega$ only, then the first set of equations (2) uniquely defines the corresponding Weyl connection $\mathbb{D}$. We will refer to $\omega$ as the Weyl covector. It was shown in [21] that, for broad classes of translationally invariant equations (1), the Weyl covector is expressed in terms of $g$ by the explicit formula

$$
\omega_{k}=2 g_{k j} \mathcal{D}_{x^{s}}\left(g^{j s}\right)+\mathcal{D}_{x^{k}}\left(\ln \operatorname{det} g_{i j}\right),
$$

where $\mathcal{D}_{x^{k}}$ denotes the total derivative with respect to $x^{k}$ :

$$
\mathcal{D}_{x^{k}}=\partial_{x^{k}}+u_{k} \partial_{u}+u_{i k} \partial_{u_{i}}+u_{i j k} \partial_{u_{i j}}+\cdots .
$$

We emphasise that in the general case formula (3) is no longer valid. Finding a universally applicable formula for the Weyl covector is one of the main objectives of this paper. Since the characteristic conformal structure $g$ depends on the 2-jet of $u$, one can show that the Weyl covector depends on no more than 3-jets, and is linear in the third-order partial derivatives of $u$. (Recall that the $k$-jet of $u$ at a point $x$ can be identified with the collection of partial derivatives $\partial^{\nu} u$ of order $|\nu| \leq k$.) 
Remark. Given a three-dimensional conformal background $[g]$, the problem of reconstruction of a covector $\omega$ such that $(g, \omega)$ satisfy the Einstein-Weyl equations is far from trivial: it was shown in $[18,19]$ that, for given $g$, EinsteinWeyl equations reduce to a complicated differential system for $\omega$. This system is overdetermined, and not in involution. Thus, it may be inconsistent (the cases of general left-invariant metric on $S^{3}$, metric 'Sol', or any 'sufficiently generic' $g$ ), or possess multiple solutions (the case of flat $g$, and some other metrics with multiple Killing vectors). In particular, there is no explicit 'formula' for $\omega$ in terms of $g$. What makes difference in our case is that we are dealing with a whole family of conformal structures parametrised by solutions $u$ of second-order PDE (1). The corresponding Einstein-Weyl equations split in the higher-order derivatives of $u$, thus providing additional constraints for $\omega$, both differential and algebraic. This leads to a formula for $\omega$ depending on the equation, and involving 3-jets of $u$ only, with the leading part given by (3).

We recall that Einstein-Weyl equations (2) are integrable by twistortheoretic methods [27]; in [12] this was explicitly demonstrated in the ManakovSantini gauge. PDEs (1) satisfying EW property can be viewed as reductions of the Einstein-Weyl equations. This, in particular, implies the existence of a dispersionless Lax representation [5]. We recall that dispersionless Lax pair consists of two parameter-dependent vector fields $\hat{X}, \hat{Y}$ for which the integrability condition

$$
[\hat{X}, \hat{Y}] \in \operatorname{span}\langle\hat{X}, \hat{Y}\rangle
$$

holds identically modulo (1). In equivalent form, dispersionless Lax pairs have first appeared in [35] as dispersionless limits of Lax pairs of integrable soliton equations. Relations of dispersionless integrable systems to Einstein-Weyl geometry have been discussed in $[4,13-17,33]$.

\subsection{Summary of Main Results}

Partial Classification Results In Sect. 2 we demonstrate that EW property is an efficient classification/integrability criterion. To illustrate the approach we obtain complete lists of PDEs with EW property within the following three classes:

- Dispersionless lattice equations

$$
u_{x y}=f\left(x, y, t, u, u_{x}, u_{y}, u_{t}, u_{t t}\right) .
$$

Modulo natural equivalence transformations preserving this class there exists a unique example with $f_{u_{t t}, u_{t t}} \neq 0$, the so-called Boyer-Finley equation $u_{x y}=e^{u_{t t}}[3]$. This example shows that EW property is a rather stringent constraint.

- Nonlinear wave equations

$$
u_{t t}=f\left(x, y, t, u, u_{x}, u_{y}, u_{t}\right) u_{x y} .
$$

The EW property leads to a generic case $f=\frac{\sinh ^{2} u_{t}}{u_{x} u_{y}}$, plus a number of degenerations. 
- Generalised Dunajski-Tod equations

$$
\left(u_{t t}-u\right) u_{x y}-\left(u_{x t}-u_{x}\right)\left(u_{y t}+u_{y}\right)=f\left(x, y, t, u, u_{x}, u_{y}, u_{t}\right) .
$$

The EW property leads to a generic case $f=c^{2} \frac{u_{x} u_{y}}{\cosh ^{2} c t}$, plus a number of degenerations.

For all equations arising in the classification we calculate the corresponding Einstein-Weyl structures and dispersionless Lax pairs. The structure of contact symmetry algebras indicates that all resulting equations are contact nonequivalent.

Reconstruction of the Weyl Covector In Sect. 3 we outline a general procedure to calculate the covector $\omega$. This procedure applies to all equations that are not of Monge-Ampère type, and gives an expression for $\omega$ in terms of the equation (see Sect. 3.1 for Monge-Ampère conditions in 3D). We look for $\omega$ in the form $\omega=\Omega+\phi$, in components,

$$
\omega_{k}=\Omega_{k}+\phi_{k}
$$

where $\Omega_{k}$ are given by formula (3) and $\phi_{k}$ are the 'correction' terms. Substituting $g, \omega$ into Einstein-Weyl conditions (2) and splitting the resulting equations in the third-order derivatives of $u$, we conclude that the correction terms $\phi_{k}$ must be functions of the 2-jet of $u$ only. Furthermore, along with a number of differential relations, $\phi_{k}$ must satisfy an algebraic system of 20 linear inhomogeneous equations which, in the non-Monge-Ampère case, determines $\phi_{k}$ uniquely.

In other words, the system has the form $A \phi=B$ where $A$ is a $20 \times 3$ matrix of rank $=3$, and $B$ is a 20 -component vector (both depend on the 2 -jet of $u$ ). Summarising, we have the following result.

Theorem 1. For every nondegenerate non-Monge-Ampère equation (1) with EW property, the Weyl covector $\omega$ is algebraically determined by the equation.

Remark. For Monge-Ampère equations the matrix of the linear system $A$ and the vector $B$ vanish identically, and further analysis is required to reconstruct $\phi_{k}$. In fact, the EW conditions provide an overdetermined differential system for $\phi$ which, in generic case, implies a formula for $\phi$ through differential closure (compatibility analysis) of the system. We demonstrate this with examples in Sect. 2 .

As a by-product of our analysis we obtain a remarkable fact that, for any second-order PDE (1) with EW property, 'freezing' the 1-jet of $u$ (that is, giving the variables $x^{i}, u, u_{i}$ arbitrary constant values), results in an integrable Hirota-type equation $F\left(u_{i j}\right)=0$.

General Formula for Dispersionless Lax Pair For equation (1) with EW property, in Sect. 4 we propose an algorithm to calculate the corresponding dispersionless Lax pair. Here is a brief summary. Let $g$ and $\omega$ be the characteristic conformal structure and the Weyl covector, respectively. Let us introduce the so-called null coframe $\theta^{0}, \theta^{1}, \theta^{2}$ such that

$$
g=4 \theta^{0} \theta^{2}-\left(\theta^{1}\right)^{2} .
$$


Let $V_{0}, V_{1}, V_{2}$ be the dual frame, and let $c_{i j}^{k}$ be the structure functions defined by commutator expansions $\left[V_{i}, V_{j}\right]=c_{i j}^{k} V_{k}$. The Lax pair is given by vector fields

$$
\hat{X}=V_{0}+\lambda V_{1}+m \partial_{\lambda}, \quad \hat{Y}=V_{1}+\lambda V_{2}+n \partial_{\lambda},
$$

where

$$
\begin{aligned}
m & =\left(\frac{1}{2} c_{12}^{1}-\frac{1}{4} \omega_{2}\right) \lambda^{3}+\left(\frac{1}{2} c_{02}^{1}-c_{12}^{2}-\frac{1}{2} \omega_{1}\right) \lambda^{2}+\left(\frac{1}{2} c_{01}^{1}-c_{02}^{2}-\frac{1}{4} \omega_{0}\right) \lambda-c_{01}^{2}, \\
n & =-c_{12}^{0} \lambda^{3}+\left(\frac{1}{2} c_{12}^{1}-c_{02}^{0}+\frac{1}{4} \omega_{2}\right) \lambda^{2}+\left(\frac{1}{2} c_{02}^{1}-c_{01}^{0}+\frac{1}{2} \omega_{1}\right) \lambda+\left(\frac{1}{2} c_{01}^{1}+\frac{1}{4} \omega_{0}\right) ;
\end{aligned}
$$

here $\omega_{i}$ are the components of the Weyl covector: $\omega=\omega_{i} \theta^{i}$. In combination with Theorem 1 we have the following result.

Theorem 2. Every nondegenerate second-order PDE with EW property is integrable, and the dispersionless Lax pair is algebraically determined by the Weyl covector $\omega$ and the function $F$ of (1).

Corollary. For every nondegenerate non-Monge-Ampère equation (1) with EW property, the dispersionless Lax pair is algebraically determined by the equation.

This result sounds, in a sense, surprising: intuition coming from the theory of soliton equations tells us that reconstruction of a Lax pair for a given PDE (known to be integrable) should require 'integration' of some kind.

Rigidity Conjecture In Sect. 5 we formulate a rigidity conjecture which states that, in the non-Monge-Ampère case, every PDE (1) with EW property can be reduced to a dispersionless Hirota form $F\left(u_{i j}\right)=0$ via a suitable contact transformation. In other words, all dependence on the 1-jet variables $x^{i}, u, u_{i}$ can be eliminated (for Monge-Ampère equations this is not true).

To illustrate this phenomenon we consider a PDE [31]

$$
u_{t t}=\frac{u_{x y}}{u_{x t}}+\frac{1}{6} \varphi\left(u_{x x}\right) u_{x t}^{2},
$$

for which EW property is equivalent to the Chazy equation $\varphi^{\prime \prime \prime}+2 \varphi \varphi^{\prime \prime}-3 \varphi^{\prime 2}=$ 0 . We prove that any deformation of the form

$$
u_{t t}=\frac{u_{x y}}{u_{x t}}+\frac{1}{6} f\left(x, u, u_{x}, u_{x x}\right) u_{x t}^{2}
$$

which satisfies EW property, is trivial (contact-equivalent to the undeformed equation). We believe that our method of proof can be extended to the general case.

\section{Examples and Classification Results}

Given a class of second-order PDEs in 3D, we impose Einstein-Weyl conditions for the characteristic conformal structure $g$ to obtain classification results. This procedure can be viewed as a 'dispersionless integrability test', and is manifestly contact-invariant. Some illustrative examples are given below. We emphasise that in all examples the Weyl covector $\omega$, as well as the associated dispersionless Lax pair, are expressible in terms of the equation by explicit formulae that work for all special cases arising in the classification. 


\subsection{Dispersionless Lattice Equations}

Here we consider equations of the form

$$
u_{x y}=f\left(x, y, t, u, u_{x}, u_{y}, u_{t}, u_{t t}\right) .
$$

In the translationally invariant case, such equations arise as dispersionless limits of integrable lattices

$$
u_{x y}^{n}=F\left(u, u_{x}, u_{y}, u^{n-1}, u^{n}, u^{n+1}\right),
$$

see [20]. The characteristic conformal structure of equation (4) has the form

$$
g=4 f_{u_{t t}} d x d y-d t^{2} .
$$

Assuming $f_{u_{t t}, u_{t t}} \neq 0$ (which is equivalent to the requirement that equation (4) does not belong to the Monge-Ampère class), one can show that the Weyl covector is given by the following formula in terms of the right-hand side $f$ :

$$
\omega=\left(\frac{2}{3} \frac{f_{u_{t}}}{f_{u_{t t}}}+\frac{10}{3} \frac{\mathcal{D}_{t}\left(f_{u_{t t}}\right)}{f_{u_{t t}}}-\frac{4}{3} \frac{\mathcal{D}_{t}\left(f_{u_{t t}, u_{t t}}\right)}{f_{u_{t t}, u_{t t}}}\right) d t
$$

where $\mathcal{D}_{t}$ denotes the total $t$-derivative. One can represent $\omega$ in the form $\omega=$ $\Omega+\phi$ where $\Omega$ as given by formula (3) and the correction term $\phi$ are as follows:

$$
\Omega=2 \frac{\mathcal{D}_{t}\left(f_{u_{t t}}\right)}{f_{u_{t t}}} d t, \quad \phi=\left(\frac{2}{3} \frac{f_{u_{t}}}{f_{u_{t t}}}+\frac{4}{3} \frac{\mathcal{D}_{t}\left(f_{u_{t t}}\right)}{f_{u_{t t}}}-\frac{4}{3} \frac{\mathcal{D}_{t}\left(f_{u_{t t}, u_{t t}}\right)}{f_{u_{t t}, u_{t t}}}\right) d t ;
$$

note the absence of $d x$ - and $d y$-components. The requirement that $g, \omega$ satisfy Einstein-Weyl conditions on every solution of equation (4) leads to a system of differential constraints (integrability conditions) for the right-hand side $f$, the simplest of them being

$$
\begin{aligned}
f_{u_{t t}, u_{t t}, u_{x}} & =\frac{f_{u_{t t}, u_{t t}} f_{u_{t t}, u_{x}}}{f_{u_{t t}}}, \quad f_{u_{t t}, u_{t t}, u_{y}}=\frac{f_{u_{t t}, u_{t t}} f_{u_{t t}, u_{y}}}{f_{u_{t t}}}, \\
f_{u_{t t}, u_{t t}, u_{t}} & =\frac{f_{u_{t t}, u_{t t}} f_{u_{t t}, u_{t}}}{f_{u_{t t}}}, \quad f_{u_{t t}, u_{x}, u_{x}}=\frac{f_{u_{t t}, u_{t t}} f_{u_{x}, u_{x}}}{f_{u_{t t}}}, \\
f_{u_{t t}, u_{x}, u_{y}} & =\frac{f_{u_{t t}, u_{x}} f_{u_{t t}, u_{y}}}{f_{u_{t t}}}, \quad f_{u_{t t}, u_{y}, u_{y}}=\frac{f_{u_{t t}, u_{t t}} f_{u_{y}, u_{y}}}{f_{u_{t t}}}, \\
f_{u_{t t}, u_{t t}, u_{t t}} & =\frac{f_{u_{t t}, u_{t t}}^{2}}{f_{u_{t t}}}, \quad f_{u_{t t}, u_{x}, u_{t}}=\frac{f_{u_{t t}, u_{x}} f_{u_{t t}, u_{t}}}{f_{u_{t t}}}, \quad f_{u_{t t}, u_{y}, u_{t}}=\frac{f_{u_{t t}, u_{y}} f_{u_{t t}, u_{t}}}{f_{u_{t t}}}, \\
f_{u_{t t}, u_{t}, u_{t}} & =\frac{f_{u_{t t}, u_{t}}^{2}}{f_{u_{t t}}}+2\left(f_{u_{t t}, u_{x}} f_{u_{t t}, u_{y}}-f_{u_{x}, u_{y}} f_{u_{t t}, u_{t t}}\right),
\end{aligned}
$$

plus a number of more complicated constraints. Note that the set of integrability conditions is not in involution, and the prolongation implies further second-order relations such as

$$
\begin{aligned}
f_{u_{t t}, u_{t t}} f_{u_{t}} & =f_{u_{t t}, u_{t}} f_{u_{t t}}, \\
f_{u_{t t}, u_{x}} & =f_{u_{t t}, u_{y}}=f_{u_{x}, u_{x}}=f_{u_{y}, u_{y}}=f_{u_{x}, u_{t}}=f_{u_{y}, u_{t}}=0, \quad \text { etc. }
\end{aligned}
$$

Consequently, modulo the equivalence transformations $u \rightarrow U(u, x, y, t)$, equation (4) has the form

$$
u_{x y}=e^{u_{t t}+\varphi u_{t}+\frac{2}{9} u\left(3 \varphi^{\prime}+\varphi^{2}\right)}
$$


where $\varphi(t)$ is an arbitrary function. It can be set equal to zero via a suitable transformation $t \rightarrow a(t), u \rightarrow b(t) u+c(t)$, thus leading to the unique canonical form

$$
u_{x y}=e^{u_{t t}}
$$

known as the Boyer-Finley (BF) equation [3]. This example demonstrates rigidity of the Einstein-Weyl requirement.

Integrable equations of type (4) possess a Lax representation $[\hat{X}, \hat{Y}] \in$ $\operatorname{span}\langle\hat{X}, \hat{Y}\rangle$ with

$$
\begin{aligned}
& \hat{X}=\partial_{y}+\lambda f_{u_{t t}} \partial_{t}+\lambda^{2}\left(\frac{2}{3} f_{u_{t t}} \frac{\mathcal{D}_{t} f_{u_{t t}, u_{t t}}}{f_{u_{t t}, u_{t t}}}-\frac{5}{3} \mathcal{D}_{t} f_{u_{t t}}-\frac{1}{3} f_{u_{t}}\right) \partial_{\lambda}, \\
& \hat{Y}=\lambda \partial_{x}+\partial_{t}+\lambda\left(\frac{1}{3} \frac{f_{u_{t}}}{f_{u_{t t}}}+\frac{2}{3} \frac{\mathcal{D}_{t} f_{u_{t t}}}{f_{u_{t t}}}-\frac{2}{3} \frac{\mathcal{D}_{t} f_{u_{t t}, u_{t t}}}{f_{u_{t t}, u_{t t}}}-\lambda \frac{\mathcal{D}_{x} f_{u_{t t}}}{f_{u_{t t}}}\right) \partial_{\lambda} .
\end{aligned}
$$

Remarkably, this Lax pair works modulo integrability conditions satisfied by $f$ and is therefore fully invariant under the equivalence transformations preserving class (4). For BF equation (5) it simplifies to

$$
\hat{X}=\partial_{y}+\lambda e^{u_{t t}} \partial_{t}-\lambda^{2} e^{u_{t t}} u_{t t t} \partial_{\lambda}, \quad \hat{Y}=\lambda \partial_{x}+\partial_{t}-\lambda^{2} u_{t t x} \partial_{\lambda} .
$$

\subsection{Nonlinear Wave Equations}

Here we consider quasilinear equations of the form

$$
u_{t t}=f\left(x, y, t, u, u_{x}, u_{y}, u_{t}\right) u_{x y} .
$$

The characteristic conformal structure is

$$
g=\frac{4}{f} d x d y-d t^{2},
$$

the corresponding Weyl covector is given by

$$
\omega=\left(-2 \mathcal{D}_{t} \ln f+\varphi(t)\right) d t .
$$

At this stage, $\varphi(t)$ is some function to be determined. One can represent $\omega$ in the form $\omega=\Omega+\phi$ where $\Omega$ as given by formula (3) and the correction term $\phi$ are as follows:

$$
\Omega=-2 \mathcal{D}_{t} \ln f d t, \quad \phi=\varphi(t) d t .
$$

The requirement that $g, \omega$ satisfy Einstein-Weyl conditions on every solution of equation (6) leads to a system of differential constraints (integrability conditions) for the right-hand side $f$. The simplest of them are as follows:

$$
\begin{aligned}
f_{u_{x}, u_{x}} & =2 \frac{f_{u_{x}}^{2}}{f}, \quad f_{u_{x}, u_{y}}=\frac{f_{u_{x}} f_{u_{y}}}{f}, \quad f_{u_{y}, u_{y}}=2 \frac{f_{u_{y}}^{2}}{f}, \\
f_{u_{x}, u_{t}} & =\frac{f_{u_{x}} f_{u_{t}}}{f}, \quad f_{u_{y}, u_{t}}=\frac{f_{u_{y}} f_{u_{t}}}{f}, \quad f_{u_{t}, u_{t}}=\frac{f f_{u_{t}}^{2}-2 f_{u_{x}} f_{u_{y}}}{f^{2}} \\
f_{u_{x}, u} & =\frac{f_{u_{x}} f_{y}+u_{y} f_{u} f_{u_{x}}-f f_{y, u_{x}}}{u_{y} f}, \quad f_{u_{y}, u}=\frac{f_{u_{y}} f_{x}+u_{x} f_{u} f_{u_{y}}-f f_{x, u_{y}}}{u_{x} f},
\end{aligned}
$$

plus four more complicated constraints that involve $\varphi(t)$. One of them is $\frac{3}{2} f_{u_{t}}\left(\varphi f-2 f_{t}-2 u_{t} f_{u}\right)+\frac{u_{x}}{u_{y}}\left(f f_{y, u_{x}}-f_{y} f_{u_{x}}\right)+\frac{u_{y}}{u_{x}}\left(f f_{x, u_{y}}-f_{x} f_{u_{y}}\right)+2 f f_{t, u_{t}}$ $+2 u_{t} f f_{u, u_{t}}+2 f_{x} f_{u_{x}}+u_{x} f_{u} f_{u_{x}}-f f_{x, u_{x}}+2 f_{y} f_{u_{y}}+u_{y} f_{u} f_{u_{y}}-f f_{y, u_{y}}=0$. 
Analysis of these constraints shows that for (nonlinear) integrable equations (6), the coefficient $f_{u_{t}}$ cannot equal zero and we obtain an explicit formula for $\varphi(t)$ in terms of $f$ :

$$
\begin{aligned}
\varphi(t)= & 2 \frac{f_{t}}{f}+2 u_{t} \frac{f_{u}}{f}-\frac{2}{3 f f_{u_{t}}} \frac{u_{x}}{u_{y}}\left(f f_{y, u_{x}}-f_{y} f_{u_{x}}\right)-\frac{2}{3 f f_{u_{t}}} \frac{u_{y}}{u_{x}}\left(f f_{x, u_{y}}-f_{x} f_{u_{y}}\right) \\
& -\frac{2}{3 f f_{u_{t}}}\left(2 f f_{t, u_{t}}+2 u_{t} f f_{u, u_{t}}+2 f_{x} f_{u_{x}}+u_{x} f_{u} f_{u_{x}}-f f_{x, u_{x}}\right. \\
& \left.+2 f_{y} f_{u_{y}}+u_{y} f_{u} f_{u_{y}}-f f_{y, u_{y}}\right) .
\end{aligned}
$$

It is a non-trivial corollary of the integrability conditions that the right-hand side of this expression is a function of $t$ only. In any case, we have an explicit formula for $\omega$ in terms of the equation.

Solving the integrability conditions results in the following generic case:

$$
u_{t t}=\frac{\sinh ^{2} u_{t}}{u_{x} u_{y}} u_{x y},
$$

as well as a number of singular strata. Normal forms are achieved modulo equivalence transformations $x \rightarrow \eta(x), y \rightarrow \psi(y), u \rightarrow u+t p(x)+t q(y)+$ $r(x)+s(y)$, translation of the $t$-variable, rescaling of $u$, discrete symmetries $x \leftrightarrow y$ and $t \mapsto-t$, and the transformation $(x, y, t, u) \mapsto(x, y, 1 / t, u / t)$, which all leave the class (6) form-invariant. The final list is summarised below.

Cases 1-6 are contact non-equivalent: while some of the symmetry algebras have the same dimensional characteristics, none are isomorphic as follows from the Lie algebra structure. In all cases $\mathfrak{g}$ is the right extension of an infinite ideal by a Lie algebra of dimension $\leq 3$ :

$$
0 \longrightarrow \mathfrak{s}_{\infty} \longrightarrow \mathfrak{g} \longrightarrow \mathfrak{s}_{\diamond} \rightarrow 0 .
$$

Below we describe $\mathfrak{g}$ via $\mathfrak{s}_{\infty}=\left\langle Z_{i}\right\rangle, \mathfrak{s}_{\diamond}=\left\langle V_{j}\right\rangle$ for each item of the table. It turns out that in all cases, $\mathfrak{s}_{\infty}$ is the derived algebra $[[\mathfrak{g}, \mathfrak{g}],[\mathfrak{g}, \mathfrak{g}]]$, and $\mathfrak{s}_{\diamond}$ is a subalgebra-complement. Thus, both the functional dimension and the number of constants are invariantly defined.

(1) The generators are $Z_{1}(a)=a(x) \partial_{x}, Z_{2}(b)=b(y) \partial_{y}, V_{1}=t \partial_{t}+u \partial_{u}$, $V_{2}=\partial_{t}, V_{3}=\partial_{u}$. Thus, $\mathfrak{s}_{\infty}=\operatorname{Vect}(\mathbb{R}) \oplus \operatorname{Vect}(\mathbb{R}), \mathfrak{s}_{\diamond}=\mathbb{R} \ltimes \mathbb{R}^{2}$ and $\left[\mathfrak{s}_{\infty}, \mathfrak{s}_{\diamond}\right]=0$.

(2) Here $Z_{1}(a)=a(x) \partial_{x}, Z_{2}(b)=b(y) \partial_{y}, Z_{3}(c)=c(u) \partial_{u}, V_{1}=t \partial_{t}, V_{2}=\partial_{t}$. Thus, $\mathfrak{s}_{\infty}=\operatorname{Vect}(\mathbb{R}) \oplus \operatorname{Vect}(\mathbb{R}) \oplus \operatorname{Vect}(\mathbb{R}), \mathfrak{s}_{\diamond}=\mathbb{R} \ltimes \mathbb{R}$ and $\left[\mathfrak{s}_{\infty}, \mathfrak{s}_{\diamond}\right]=0$.

(3) Here $Z_{1}(a)=a(x) \partial_{x}+a^{\prime}(x) t \partial_{u}, Z_{2}(b)=b(x) \partial_{u}, Z_{3}(c)=c(y) \partial_{y}$, $V_{1}=x \partial_{x}+t \partial_{t}+u \partial_{u}, V_{2}=\partial_{t}$. Thus, $\mathfrak{s}_{\infty}=\mathfrak{s}_{\infty}^{\prime} \oplus \mathfrak{s}_{\infty}^{\prime \prime}$ with $\mathfrak{s}_{\infty}^{\prime}=\operatorname{Vect}(\mathbb{R}) \ltimes$ $C^{\infty}(\mathbb{R})$ and $\mathfrak{s}_{\infty}^{\prime \prime}=\operatorname{Vect}(\mathbb{R}), \mathfrak{s}_{\diamond}=\mathbb{R} \ltimes \mathbb{R}$ and in addition $\left[\mathfrak{s}_{\infty}, \mathfrak{s}_{\diamond}\right]=\mathfrak{s}_{\infty}^{\prime}$.

(4) Here $Z_{1}(a)=a(x) \partial_{x}-\left(a^{\prime}(x) u+\frac{1}{2} t^{2} a^{\prime \prime}(x)\right) \partial_{u}, Z_{2}(b)=b(x) \partial_{u}, Z_{3}(c)=$ $c(x) t \partial_{u}, Z_{4}(d)=d(y) \partial_{y}, V_{1}=t \partial_{t}+2 u \partial_{u}, V_{2}=\partial_{t}$. Thus, $\mathfrak{s}_{\infty}=\mathfrak{s}_{\infty}^{\prime} \oplus \mathfrak{s}_{\infty}^{\prime \prime}$ with $\mathfrak{s}_{\infty}^{\prime}=\operatorname{Vect}(\mathbb{R}) \ltimes\left(C^{\infty}(\mathbb{R}) \oplus C^{\infty}(\mathbb{R})\right)$ and $\mathfrak{s}_{\infty}^{\prime \prime}=\operatorname{Vect}(\mathbb{R}), \mathfrak{s}_{\diamond}=\mathbb{R} \ltimes \mathbb{R}$ and in addition $\left[\mathfrak{s}_{\infty}, \mathfrak{s}_{\diamond}\right]=C^{\infty}(\mathbb{R}) \oplus C^{\infty}(\mathbb{R}) \subset \mathfrak{s}_{\infty}^{\prime}$.

(5) Here $Z_{1}(a)=a(x) \partial_{x}+a^{\prime}(x) t \partial_{u}, Z_{2}(b)=b(x) \partial_{u}, Z_{3}(c)=c(y) \partial_{y}+$ $c^{\prime}(y) t \partial_{u}, Z_{4}(d)=d(y) \partial_{u}, V_{1}=t \partial_{t}+\left(u-\frac{1}{2} t\right) \partial_{u}$. Thus, $\mathfrak{s}_{\infty}=\mathfrak{s}_{\infty}^{\prime} \oplus$ 


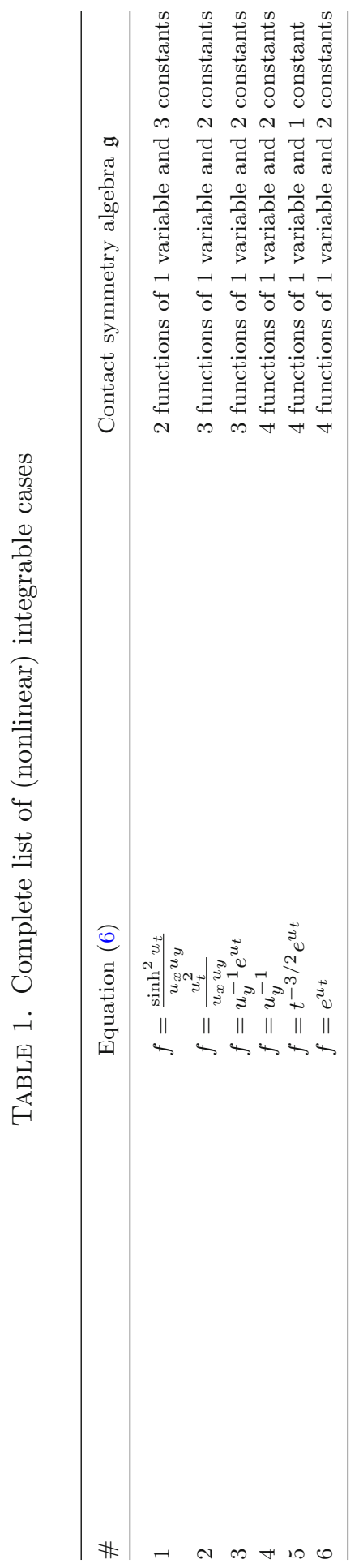


$\mathfrak{s}_{\infty}^{\prime \prime} /\left\langle Z_{2}(1)=Z_{4}(1)\right\rangle$ with $\mathfrak{s}_{\infty}^{\prime}=\operatorname{Vect}(\mathbb{R}) \ltimes C^{\infty}(\mathbb{R})=\mathfrak{s}_{\infty}^{\prime \prime}, \mathfrak{s}_{\diamond}=\mathbb{R}$ and $\left[\mathfrak{s}_{\infty}, \mathfrak{s}_{\diamond}\right]=C^{\infty}(\mathbb{R})^{\prime} \oplus C^{\infty}(\mathbb{R})^{\prime \prime} \subset \mathfrak{s}_{\infty}$.

(6) This has the same $\mathfrak{s}_{\infty}$ as in case (5), but $\mathfrak{s}_{\diamond}=\mathbb{R} \ltimes \mathbb{R}$ is generated by $V_{1}=2 y \partial_{y}+t \partial_{t}+u \partial_{u}, V_{2}=\partial_{t}$. In addition $\left[\mathfrak{s}_{\infty}, \mathfrak{s}_{\diamond}\right]=C^{\infty}(\mathbb{R})^{\prime} \oplus \mathfrak{s}_{\infty}^{\prime \prime}$.

Equations from Table 1 possess a Lax representation $[\hat{X}, \hat{Y}] \in \operatorname{span}\langle\hat{X}, \hat{Y}\rangle$ with

$\hat{X}=f \partial_{y}+\lambda \partial_{t}+\lambda^{2}\left(\mathcal{D}_{t} \log f-\frac{1}{2} \varphi\right) \partial_{\lambda}, \quad \hat{Y}=\lambda \partial_{x}+\partial_{t}+\lambda\left(\lambda \mathcal{D}_{x} \log f+\frac{1}{2} \varphi\right) \partial_{\lambda}$,

here $\varphi(t)$ is the same as in the formula for the Weyl covector. Note that this Lax pair works for all cases from Table 1 (upon substitution of the corresponding expression for $f$ ). In fact, one can say more: this Lax pair works modulo the integrability conditions satisfied by $f$, that is, it is invariant under the equivalence transformations used to obtain cases $1-6$.

Remark. Contact symmetry algebra of the BF equation, $u_{x y}=e^{u_{t t}}$, contains 6 functions of 1 variable; therefore, it is not equivalent to any of the items in Table 1. Indeed, for the BF equation we have: $Z_{1}(a)=a(x) \partial_{x}+\frac{1}{2} a^{\prime}(x) t^{2} \partial_{u}$, $Z_{2}(b)=b(x) t \partial_{u}, Z_{3}(c)=c(x) \partial_{u}, Z_{4}(d)=d(y) \partial_{y}+\frac{1}{2} d^{\prime}(y) t^{2} \partial_{u}, Z_{5}(e)=$ $e(y) t \partial_{u}, Z_{6}(f)=f(y) \partial_{u}, V_{1}=t \partial_{t}+2 u \partial_{u}, V_{2}=\partial_{t}$. Therefore, we have $\mathfrak{s}_{\infty}=\mathfrak{s}_{\infty}^{\prime} \oplus \mathfrak{s}_{\infty}^{\prime \prime}$ where $\mathfrak{s}_{\infty}^{\prime}=\operatorname{Vect}(\mathbb{R}) \ltimes\left(C^{\infty}(\mathbb{R}) \oplus C^{\infty}(\mathbb{R})\right)=\mathfrak{s}_{\infty}^{\prime \prime} ; \mathfrak{s}_{\diamond}=\operatorname{sol}(2)$ and $\left[\mathfrak{s}_{\infty}, \mathfrak{s}_{\diamond}\right]=\left(C^{\infty}(\mathbb{R}) \oplus C^{\infty}(\mathbb{R})\right)^{\prime} \oplus\left(C^{\infty}(\mathbb{R}) \oplus C^{\infty}(\mathbb{R})\right)^{\prime \prime}$. Note though that $\mathrm{BF}$ equation can be obtained by potentiation from case 6 of Table 1 .

\subsection{Generalised Dunajski-Tod Equations}

Here we consider Monge-Ampère equations of the form

$$
\left(u_{t t}-u\right) u_{x y}-\left(u_{x t}-u_{x}\right)\left(u_{y t}+u_{y}\right)=f\left(x, y, t, u, u_{x}, u_{y}, u_{t}\right) .
$$

For $f=4 e^{2 \rho t}$ this equation was discussed by Dunajski and Tod in the context of hyper-Kähler metrics with conformal symmetry [16]. The characteristic conformal structure of equation (7) has the form

$$
g=\left(u d t+u_{x} d x-u_{y} d y-d u_{t}\right)^{2}+4 f d x d y .
$$

One can show that the Weyl covector can be expressed in terms of the righthand side $f$ :

$$
\omega=2\left(\frac{u_{x t}-u_{x}}{u_{t t}-u} d x-\frac{u_{y t}+u_{y}}{u_{t t}-u} d y\right)+2 R\left(d t+\frac{u_{x t}-u_{x}}{u_{t t}-u} d x+\frac{u_{y t}+u_{y}}{u_{t t}-u} d y\right),
$$

where $R=\frac{\mathcal{D}_{t} f}{f}$. For $f=4 e^{2 \rho t}$ we have $R=2 \rho$, which results in the EinsteinWeyl structure from [16]. The first term in formula (8) coincides with $\Omega$ as given by (3), the second term is the correction $\phi$. The requirement that $g, \omega$ satisfy Einstein-Weyl conditions on every solution of equation (7) leads to a system of differential constraints (integrability conditions) for the right-hand side $f$. The simplest of them are as follows:

$$
f_{u_{x}, u_{x}}=0, \quad f_{u_{x}, u_{y}} f-f_{u_{x}} f_{u_{y}}=0, \quad f_{u_{y}, u_{y}}=0,
$$


plus a number of more complicated constraints. Solving the integrability conditions results in the generic case

$$
\left(u_{t t}-u\right) u_{x y}-\left(u_{x t}-u_{x}\right)\left(u_{y t}+u_{y}\right)=c^{2} \frac{u_{x} u_{y}}{\cosh ^{2} c t}
$$

(where $c$ is an arbitrary constant), as well as a number of other strata. Normal forms are obtained modulo the following equivalence transformations: $(x, y, t, u) \mapsto\left(\eta(x), \psi(y), t, u+p(x) e^{t}+q(y) e^{-t}\right)$, rescaling of $u$, translations of the $t$-variable, and discrete symmetry $(x, y, t, u) \mapsto(y, x,-t, u)$, which all leave the class (7) form-invariant. Thus we obtain the following integrable cases:

$$
\begin{aligned}
& f=c^{2} \frac{\left(u_{x}+u_{t}+u\right)\left(u_{y}+u_{t}-u\right)}{\cosh ^{2} c(x+y-t)}, \quad f=c^{2} \frac{\left(u_{x}+u_{t}+u\right) u_{y}}{\cosh ^{2} c(x-t)}, \\
& f=c^{2} \frac{u_{x} u_{y}}{\cosh ^{2} c t}, \quad f=\frac{\left(u_{t}+u\right)\left(u_{t}-u\right)}{(x-y)^{2}}, \\
& f=e^{c t}\left(x u_{x}-\frac{u_{t}+u}{c+1}\right), \quad f=e^{-t}\left(u_{x}+u_{t}+u\right), \\
& f=e^{c t} u_{x}, \quad f=e^{t}\left(u_{t}-u\right), \quad f=e^{c t} .
\end{aligned}
$$

This list can be reduced further via point transformations as follows.

$$
\begin{aligned}
& (x, y, t, u) \mapsto\left(\frac{1}{2} e^{2 x},-\frac{1}{2} e^{-2 y}, t-x-y, u e^{x-y}\right) \text { maps } f=c^{2} \frac{\left(u_{x}+u_{t}+u\right)\left(u_{y}+u_{t}-u\right)}{\cosh ^{2} c(x+y-t)} \\
& \text { to } f=c^{2} \frac{u_{x} u_{y}}{\cosh ^{2} c t}, \\
& (x, y, t, u) \mapsto\left(\frac{1}{2} e^{2 x}, y, t-x, u e^{x}\right) \text { maps } f=c^{2} \frac{\left(u_{x}+u_{t}+u\right) u_{y}}{\cosh ^{2} c(x-t)} \text { to } f=c^{2} \frac{u_{x} u_{y}}{\cosh ^{2} c t}, \\
& (x, y, t, u) \mapsto\left(\frac{x^{(c-1) /(c+1)}}{(c-1)}, y, \frac{t+\ln (x)}{(c+1)}, u x^{-1 /(c+1)}\right) \text { maps } f=e^{c t}\left(x u_{x}-\right. \\
& \left.\frac{u_{t}+u}{c+1}\right) \text { to } f=e^{c t} u_{x} .
\end{aligned}
$$

The final list of integrable cases is summarised below.

Cases 1-6 are contact non-equivalent: this follows from the structure of their contact symmetry algebras, where we use the notation of Sect. 2.2.

(1) The generators are $Z_{1}(a)=a(x) \partial_{x}, Z_{2}(b)=b(y) \partial_{y}, V_{1}=e^{t} \partial_{u}$, $V_{2}=e^{-t} \partial_{u}, V_{3}=u \partial_{u}$. Thus, $\mathfrak{s}_{\infty}=\operatorname{Vect}(\mathbb{R}) \oplus \operatorname{Vect}(\mathbb{R}), \mathfrak{s}_{\diamond}=\mathbb{R} \ltimes \mathbb{R}^{2}$ and $\left[\mathfrak{s}_{\infty}, \mathfrak{s}_{\diamond}\right]=0$.

(2) Here $Z_{1}(a)=a(x)\left(\partial_{t}-u \partial_{u}\right), Z_{2}(b)=b(y)\left(\partial_{t}+u \partial_{u}\right), V_{1}=\partial_{x}+\partial_{y}$, $V_{2}=x \partial_{x}+y \partial_{y}, V_{3}=x^{2} \partial_{x}+y^{2} \partial_{y}$. Thus, $\mathfrak{s}_{\infty}=C^{\infty}(\mathbb{R}) \oplus C^{\infty}(\mathbb{R})$, $\mathfrak{s}_{\diamond}=\mathfrak{s l}(2)$ and $\left[\mathfrak{s}_{\infty}, \mathfrak{s}_{\diamond}\right]=\mathfrak{s}_{\infty}$.

(3) Here $Z_{1}(a)=a(x) \partial_{x}, Z_{2}(b)=b(y) \partial_{y}-\frac{b^{\prime}(y)}{c-1}\left(\partial_{t}+u \partial_{u}\right), Z_{3}(c)=$ $c(y) e^{-t} \partial_{u}, V_{1}=\partial_{t}-c y \partial_{y}, V_{2}=e^{t} \partial_{u}$. Thus, $\mathfrak{s}_{\infty}=\mathfrak{s}_{\infty}^{\prime} \oplus \mathfrak{s}_{\infty}^{\prime \prime}$, where $\mathfrak{s}_{\infty}^{\prime}=\operatorname{Vect}(\mathbb{R})$ and $\mathfrak{s}_{\infty}^{\prime \prime}=\operatorname{Vect}(\mathbb{R}) \ltimes C^{\infty}(\mathbb{R}) ; \mathfrak{s}_{\diamond}=\mathbb{R} \ltimes \mathbb{R}=\operatorname{sol}(2)$. In addition, $\left[\mathfrak{s}_{\infty}, \mathfrak{s}_{\diamond}\right]=\mathfrak{s}_{\infty}^{\prime \prime}$ for $c \neq 0$ and $\left[\mathfrak{s}_{\infty}, \mathfrak{s}_{\diamond}\right]=C^{\infty}(\mathbb{R}) \subset \mathfrak{s}_{\infty}^{\prime \prime}$ for $c=0$. $\left(3^{+}\right)$Here $Z_{1}(a)=a(x) \partial_{x}, Z_{2}(b)=b(y)\left(\partial_{t}+u \partial_{u}\right), Z_{3}(c)=c(y) e^{-t} \partial_{u}$, $V_{1}=y \partial_{y}-\partial_{t}, V_{2}=\partial_{y}, V_{3}=e^{t} \partial_{u}$. Thus, $\mathfrak{s}_{\infty}=\operatorname{Vect}(\mathbb{R}) \oplus\left(C^{\infty}(\mathbb{R}) \ltimes\right.$ $\left.C^{\infty}(\mathbb{R})\right) ; \mathfrak{s}_{\diamond}=\mathbb{R} \ltimes \mathbb{R}^{2}$ and $\left[\mathfrak{s}_{\infty}, \mathfrak{s}_{\diamond}\right]=C^{\infty}(\mathbb{R}) \ltimes C^{\infty}(\mathbb{R})$.

(4) Here $Z_{1}(a)=a(x)\left(\partial_{x}+\partial_{t}-u \partial_{u}\right), Z_{2}(b)=b(y) \partial_{y}+\frac{1}{2} b^{\prime}(y)\left(\partial_{t}+u \partial_{u}\right)$, $Z_{3}(c)=c(y) e^{-t} \partial_{u}, V_{1}=\partial_{x}, V_{2}=e^{t-2 x} \partial_{u}$. Thus, $\mathfrak{s}_{\infty}=\mathfrak{s}_{\infty}^{\prime} \oplus \mathfrak{s}_{\infty}^{\prime \prime}$, where $\mathfrak{s}_{\infty}^{\prime}=\operatorname{Vect}(\mathbb{R})$ and $\mathfrak{s}_{\infty}^{\prime \prime}=\operatorname{Vect}(\mathbb{R}) \ltimes C^{\infty}(\mathbb{R}) ; \mathfrak{s}_{\diamond}=\operatorname{sol}(2)$ and $\left[\mathfrak{s}_{\infty}, \mathfrak{s}_{\diamond}\right]=\mathfrak{s}_{\infty}^{\prime}$. 


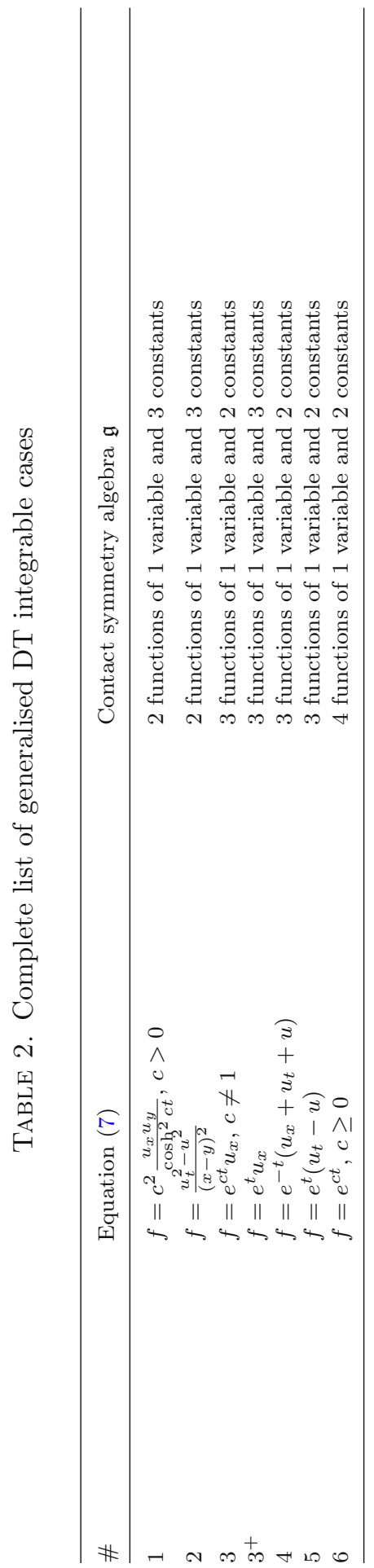


(5) Here $Z_{1}(a)=a(x) \partial_{x}-\frac{1}{2} a^{\prime}(x)\left(\partial_{t}-u \partial_{u}\right), Z_{2}(b)=b(x) e^{t} \partial_{u}, Z_{3}(c)=$ $c(y)\left(\partial_{t}+u \partial_{u}\right), V_{1}=y \partial_{y}-\partial_{t}, V_{2}=\partial_{y}$. Thus, $\mathfrak{s}_{\infty}=\mathfrak{s}_{\infty}^{\prime} \oplus \mathfrak{s}_{\infty}^{\prime \prime}$, where $\mathfrak{s}_{\infty}^{\prime}=$ $\operatorname{Vect}(\mathbb{R}) \ltimes C^{\infty}(\mathbb{R}), \mathfrak{s}_{\infty}^{\prime \prime}=C^{\infty}(\mathbb{R}) ; \mathfrak{s}_{\diamond}=\operatorname{sol}(2)$ and $\left[\mathfrak{s}_{\infty}, \mathfrak{s}_{\diamond}\right]=C^{\infty}(\mathbb{R})^{\prime} \oplus$ $C^{\infty}(\mathbb{R})^{\prime \prime}$.

$(6: c \neq 2)$ Here $Z_{1}(a)=a(x) \partial_{x}-\frac{a^{\prime}(x)}{c+2}\left(\partial_{t}-u \partial_{u}\right), Z_{2}(b)=b(x) e^{t} \partial_{u}$, $Z_{3}(c)=c(y) \partial_{y}-\frac{c^{\prime}(y)}{c-2}\left(\partial_{t}+u \partial_{u}\right), Z_{4}(d)=d(y) e^{-t} \partial_{u}, V_{1}=x \partial_{x}-y \partial_{y}$, $V_{2}=\partial_{t}+\frac{c}{2} u \partial_{u}$. Thus, $\mathfrak{s}_{\infty}=\mathfrak{s}_{\infty}^{\prime} \oplus \mathfrak{s}_{\infty}^{\prime \prime}$, where $\mathfrak{s}_{\infty}^{\prime}=\operatorname{Vect}(\mathbb{R}) \ltimes C^{\infty}(\mathbb{R})=$ $\mathfrak{s}_{\infty}^{\prime \prime} ; \mathfrak{s}_{\diamond}=\mathbb{R}^{2}$ and $\left[\mathfrak{s}_{\infty}, \mathfrak{s}_{\diamond}\right]=\mathfrak{s}_{\infty}$.

$(6: c=2)$ Here $Z_{1}(a)=a(x) \partial_{x}-\frac{a^{\prime}(x)}{4}\left(\partial_{t}-u \partial_{u}\right), Z_{2}(b)=b(x) e^{t} \partial_{u}$, $Z_{3}(c)=c(y)\left(\partial_{t}+u \partial_{u}\right), Z_{4}(d)=d(y) e^{-t} \partial_{u}, V_{1}=y \partial_{y}-x \partial_{x}, V_{2}=\partial_{y}$. Thus, $\mathfrak{s}_{\infty}=\mathfrak{s}_{\infty}^{\prime} \oplus \mathfrak{s}_{\infty}^{\prime \prime}$, where $\mathfrak{s}_{\infty}^{\prime}=\operatorname{Vect}(\mathbb{R}) \ltimes C^{\infty}(\mathbb{R}), \mathfrak{s}_{\infty}^{\prime \prime}=C^{\infty}(\mathbb{R}) \ltimes$ $C^{\infty}(\mathbb{R}) ; \mathfrak{s}_{\diamond}=\operatorname{sol}(2)$ and $\left[\mathfrak{s}_{\infty}, \mathfrak{s}_{\diamond}\right]=\mathfrak{s}_{\infty}$.

Items 3 and 6 contain a parameter $c$ which is uniquely characterised by the structure equations.

Item 1 also contains a parameter $c$, yet it does not enter the structure equations. In this case non-equivalence does not follow from the symmetry analysis. Instead, we consider (point) transformations inducing an automorphism of the symmetry algebra and preserving the orbit structure. It is easy to see that such transformations, leaving the class of Dunajski-Tod equations (7) form-invariant, are only $(x, y, t, u) \mapsto(X(x), Y(y), t, k u)$ and so cannot change $c$. Thus, the parameter $c$ is essential.

Remark 1. A comparison between the two tables shows that a possible isomorphism may exist for the following two cases:

- Table 1(1) to Table 2(1). The symmetry algebras are abstractly isomorphic, yet the corresponding two-dimensional subalgebras $\left[\mathfrak{s}_{\diamond}, \mathfrak{s}_{\diamond}\right]$ have orbits of dimensions 2 and 1, respectively, hence the items are not equivalent.

- Table $1(3)$ to Table $2(3)(c \neq 0)$. The symmetry algebras are abstractly isomorphic, yet the corresponding infinite-dimensional subalgebras $\mathfrak{s}_{\infty}^{\prime}$ have orbits of dimensions 2 and 1, respectively, hence the items are not equivalent.

Thus, all integrable equations from Sect. 2 (Tables 1-2 and BF equation) are pairwise contact non-equivalent.

Remark 2. For items $2,3^{+}, 5,6(c=2)$ of Table 2 , the infinite part of the symmetry algebra is not perfect: $\left[\mathfrak{s}_{\infty}, \mathfrak{s}_{\infty}\right] \varsubsetneqq \mathfrak{s}_{\infty}$. Yet a closer analysis shows that the splitting and the numerical characteristics of Table 2 are invariantly defined.

Remark 3. The generalised Dunajski-Tod equation is quasi-linearisable: the contact transformation

$$
\Phi\left(x, y, t, u, u_{x}, u_{y}, u_{t}\right)=\left(x, y, \frac{1}{2} \ln u_{t}, \frac{u-t u_{t}}{\sqrt{u_{t}}}, \frac{u_{x}}{\sqrt{u_{t}}}, \frac{u_{y}}{\sqrt{u_{t}}}, \frac{-u-t u_{t}}{\sqrt{u_{t}}}\right)
$$


maps equation (7) to the quasilinear equation

$$
u_{x} u_{y t}-u_{t} u_{x y}=h\left(x, y, t, u, u_{x}, u_{y}, u_{t}\right) u_{t t}
$$

where $h=\frac{1}{4} \Phi^{*}(f)$. Equation (9) can be viewed as a deformation of the Bogdanov equation [1]. In the case $f=4 e^{2 \rho t}$ considered by Dunajski-Tod [16], equation (9) becomes the integrable PDE studied in [1]:

$$
u_{x} u_{y t}-u_{t} u_{x y}=u_{t}^{\rho} u_{t t} .
$$

The conformal structure for equation (9) is represented by the metric

$$
g=4 h d x d y+u_{t}^{-1}\left(u_{x} d x+u_{t} d t\right)^{2},
$$

and the corresponding Weyl covector is given by the formula

$$
\omega=\left(2 \frac{u_{x}}{u_{t}} \mathcal{D}_{t} \ln h-\mathcal{D}_{t} \frac{u_{x}}{u_{t}}\right) d x+\mathcal{D}_{t} \ln u_{y} d y+2 \mathcal{D}_{t} \ln h d t .
$$

Note that Weyl covector (10) satisfies formula (3), i.e. no 'correction' is required, while the covector $\omega$ for generalised Dunajski-Tod equation (7) does not satisfy (3), with the 'correction' being the second term containing $R$ in (8). This demonstrates contact non-invariance of formula (3), while the covector $\omega=\Omega+\phi$ given by Theorem 1 is genuinely contact invariant.

The dispersionless Lax pairs for both generalised Dunajski-Tod (7) and generalised Bogdanov (9) equations can be obtained by the recipe from the proof of Theorem 2. For the former, see Example 3 of $\S 4.2$. This implies the Lax pair for the latter via the contact transformation $\Phi$.

\section{Reconstruction of the Weyl Covector}

We begin by describing the constraints for a PDE to be of Monge-Ampère type.

\subsection{The Monge-Ampère Property}

Recall that equation (1) is said to be of Monge-Ampère type if its left-hand side can be represented as a linear combination of minors (of all possible orders) of the Hessian matrix of the function $u$ (with coefficients depending on the 1 -jet of $u$ ). Let us represent equation (1) in evolutionary form

$$
u_{00}=f\left(x^{0}, x^{1}, x^{2}, u, u_{0}, u_{1}, u_{2}, u_{01}, u_{02}, u_{11}, u_{12}, u_{22}\right) .
$$

To calculate the Weyl covector, we will need explicit differential constraints for the right-hand side $f$ that are equivalent to the Monge-Ampère property. These have only been known in low dimensions $[2,11,26,32]$. In full generality, they were obtained recently in [23]. In 3D, the Monge-Ampère conditions consist of two groups of equations for $f$. First of all, for every $i \in\{1,2\}$ one has the relations

$$
f_{u_{i i}} f_{u_{0 i} u_{0 i}}+f_{u_{i i} u_{i i}}=0, f_{u_{0 i}} f_{u_{0 i} u_{0 i}}+2 f_{u_{0 i} u_{i i}}=0 .
$$


Secondly, for every pair of distinct indices $i \neq j \in\{1,2\}$ one has the relations

$$
\begin{gathered}
f_{u_{0 j}} f_{u_{0 i} u_{0 i}}+2 f_{u_{0 i}} f_{u_{0 i} u_{0 j}}+2 f_{u_{0 i} u_{i j}}+2 f_{u_{0 j} u_{i i}}=0 \\
f_{u_{i j}} f_{u_{0 i} u_{0 i}}+2 f_{u_{i i}} f_{u_{0 i} u_{0 j}}+2 f_{u_{i i} u_{i j}}=0 \\
f_{u_{j j}} f_{u_{0 i} u_{0 i}}+f_{u_{i i}} f_{u_{0 j} u_{0 j}}+2 f_{u_{i j}} f_{u_{0 i} u_{0 j}}+2 f_{u_{i i} u_{j j}}+f_{u_{i j} u_{i j}}=0 .
\end{gathered}
$$

Due to the contact invariance of the Monge-Ampère class, the system of nine relations (12)-(13) is invariant under arbitrary contact transformations.

\subsection{Proof of Theorem 1}

Let us consider a second-order PDE in evolutionary form (11). Note that if a particular equation under study is not evolutionary, it can be brought to evolutionary form via a suitable linear change of the independent variables.

It will be convenient to rewrite Einstein-Weyl conditions (2) in terms of the Levi-Civita connection of the conformal structure $g$ (choose any representative of the conformal class):

$$
r_{i j}+\frac{1}{2}\left(\nabla_{i} \omega_{j}+\nabla_{j} \omega_{i}\right)-\frac{1}{4} \omega_{i} \omega_{j}=\Lambda g_{i j}
$$

where $\nabla$ denotes covariant differentiation in the Levi-Civita connection of $g$, and $r_{i j}$ is the corresponding Ricci tensor (which is automatically symmetric), see [28]. Since $g$ depends on the 2-jet of the function $u$, the Ricci tensor $r_{i j}$ depends on the 4 -jet of $u$. This implies that components $\omega_{k}$ must depend on the 3 -jet of $u$, furthermore, the dependence of $\omega_{k}$ on the third-order derivatives of $u$ must be affine. Analysis of the dependence of the left-hand side of (14) on the fourth-order derivatives of $u$ suggests a substitution

$$
\omega_{k}=\Omega_{k}+\phi_{k}
$$

where $\Omega_{k}$ is given by formula (3), and the 'correction terms' $\phi_{k}$ are some functions to be determined (we will see that they can only depend on the 2 -jet of $u$ ). Under this substitution equations (14) take the form

$$
\begin{aligned}
& r_{i j}+\frac{1}{2}\left(\nabla_{i} \Omega_{j}+\nabla_{j} \Omega_{i}\right)+\frac{1}{2}\left(\nabla_{i} \phi_{j}+\nabla_{j} \phi_{i}\right)-\frac{1}{4} \Omega_{i} \Omega_{j} \\
& -\frac{1}{4}\left(\Omega_{i} \phi_{j}+\Omega_{j} \phi_{i}\right)-\frac{1}{4} \phi_{i} \phi_{j}=\Lambda g_{i j} .
\end{aligned}
$$

Let us denote by $S$ the system obtained from (16) by eliminating $\Lambda$ and restricting the resulting five equations to solutions of PDE (11), that is, reducing the result modulo (11) and its differential prolongation. Equations of system $S$ possess terms of several different types: (a) linear in the fourth-order derivatives of $u$, (b) quadratic in the third-order derivatives of $u$, (c) linear in the third-order derivatives of $u$, and (d) depending on the 2-jet of $u$ only. We will discuss them case-by-case below.

(a) Terms Linear in the Fourth-Order Derivatives of $u$ There are two sources of such terms: expressions $r_{i j}+\frac{1}{2}\left(\nabla_{i} \Omega_{j}+\nabla_{j} \Omega_{i}\right)$ and $\frac{1}{2}\left(\nabla_{i} \phi_{j}+\nabla_{j} \phi_{i}\right)$. Direct calculation shows that all terms with fourth-order derivatives of $u$ coming from the expressions $r_{i j}+\frac{1}{2}\left(\nabla_{i} \Omega_{j}+\nabla_{j} \Omega_{i}\right)$ cancel out. Thus, the only source of such terms is expressions $\frac{1}{2}\left(\nabla_{i} \phi_{j}+\nabla_{j} \phi_{i}\right)$, and this implies that $\phi_{k}$ must be functions 
of the 2-jet of $u$ only: $\phi_{k}=\phi_{k}\left(x^{0}, x^{1}, x^{2}, u, u_{0}, u_{1}, u_{2}, u_{01}, u_{02}, u_{11}, u_{12}, u_{22}\right)$, recall that $u_{00}$ can be eliminated via (11). In other words, ansatz (15) captures the dependence of $\omega$ on the third-order derivatives of $u$. For several classes of (translationally invariant) second-order PDEs the terms $\phi_{k}$ vanish identically; however, they are not zero in general. Under conformal rescalings $g \rightarrow \lambda g$ both covectors $\omega$ and $\Omega$ transform as $\omega \rightarrow \omega+d \ln \lambda, \Omega \rightarrow \Omega+d \ln \lambda$. Thus, the covector $\phi=\phi_{k} d x^{k}$ is invariant with respect to conformal rescalings.

(b) Terms Quadratic in the Third-Order Derivatives of $u$ Such terms come from the expressions $r_{i j}+\frac{1}{2}\left(\nabla_{i} \Omega_{j}+\nabla_{j} \Omega_{i}\right)-\frac{1}{4} \Omega_{i} \Omega_{j}$, and do not involve $\phi_{k}$. Equating to zero the corresponding coefficients we obtain all third-order partial derivatives of the function $f$ with respect to the variables $u_{01}, u_{02}, u_{11}, u_{12}, u_{22}$, which identically coincide with the integrability conditions for Hirota-type equations

$$
u_{00}=f\left(u_{01}, u_{02}, u_{11}, u_{12}, u_{22}\right)
$$

obtained in [24]. This leads to a somewhat surprising conclusion: taking an integrable equation (11) and 'freezing' the 1-jet of $u$ (that is, giving the variables $x^{0}, x^{1}, x^{2}, u, u_{0}, u_{1}, u_{2}$ arbitrary constant values), we obtain an integrable Hirota-type equation. Note that the generic integrable Hirota type equation is a highly transcendental object: it coincides with the equation of the genus three hyperelliptic divisor [10].

(c) Terms Linear in the Third-Order Derivatives of $u$ These terms come from the expressions $r_{i j}+\frac{1}{2}\left(\nabla_{i} \Omega_{j}+\nabla_{j} \Omega_{i}\right)+\frac{1}{2}\left(\nabla_{i} \phi_{j}+\nabla_{j} \phi_{i}\right)-\frac{1}{4} \Omega_{i} \Omega_{j}-\frac{1}{4}\left(\Omega_{i} \phi_{j}+\Omega_{j} \phi_{i}\right)$. Each of the five equations of system $S$ has seven terms linear in the third-order derivatives $u_{011}, u_{012}, u_{022}, u_{111}, u_{112}, u_{122}, u_{222}$, recall that we work modulo (11) and its differential prolongation. Equating the corresponding coefficients to zero gives 35 relations involving $\phi_{k}$ and their first-order derivatives with respect to $u_{01}, u_{02}, u_{11}, u_{12}, u_{22}$. Eliminating the derivatives of $\phi_{k}$ we obtain a system of 20 equations which are linear inhomogeneous in $\phi_{k}$

(we do not write the equations explicitly due to their complexity). It is exactly at this step that we can determine $\phi$ (and hence $\omega$ ) in terms of the function $f$. It should be stressed that the linear system of 20 equations for $\phi_{k}$ is nontrivial only if equation (11) is not of Monge-Ampère type: in this case the linear system can be represented in matrix form $A \phi=B$ where $\phi=\left(\phi_{0}, \phi_{1}, \phi_{2}\right)^{T}, B$ is a vector with 20 components and $A$ is a $20 \times 3$ matrix, whose coefficients depend linearly on the left-hand sides of the Monge-Ampère conditions (12)-(13). For equations of non-Monge-Ampère type the unknowns $\phi_{k}$ can be reconstructed uniquely because $A$ necessarily contains a nonzero $3 \times 3$ minor. This is equivalent to the condition $\operatorname{rank}(A)=3$, note that we do not require $\operatorname{rk}(A \mid B)=3$ as in the Cramer rule. Indeed, the entire set of EW conditions (more precisely, the differential closure of this system) decomposes into constraints on $\phi_{k}$ and equations not containing $\phi_{k}$; the latter are integrability conditions for (11). Thus, part of the constraints $A \phi=B$ contributes to the integrability conditions for the function $f$.

(d) Terms Depending on the 2-jet of $u$ For Monge-Ampère equations, both the matrix $A$ and the vector $B$ of the linear system $A \phi=B$ vanish identically. 
In this case further analysis is required. Constraints of the second order in $u$ involve the derivatives of $\phi_{k}$; this overdetermined system for $\phi$ is not in involution. Generically, the differential closure provides more PDEs that can ultimately lead to algebraic formulae for $\phi$ via a finite jet of $u$. Numerous examples show that this is indeed the case, and that the Weyl covector $\omega$ can be reconstructed in terms of the equation even for generic Monge-Ampère equations. However, explicit conditions and demonstration of this are outside the scope of our paper.

This finishes the proof of Theorem 1.

Calculations described above to reconstruct the Weyl covector $\omega$ are implemented in a Mathematica program which is available from the archive version of this paper, arXiv:2104.02716.

\subsection{Examples of Computations}

To illustrate the general procedure, let us go through steps (a)-(c) for the two particular classes.

Example 1. Let us begin with equations

$$
u_{t t}=f\left(x, y, t, u, u_{x}, u_{y}, u_{t}, u_{x y}\right)
$$

the evolutionary form of lattice equations from Sect. 2.1. The characteristic conformal structure is ( $\left.\operatorname{set} b=u_{x y}\right)$ :

$$
g=4 d x d y-f_{b} d t^{2}
$$

We will assume $f_{b b} \neq 0$; otherwise the equation is of Monge-Ampère type.

Step (a): calculation of $\Omega$ using formula (3) gives

$$
\Omega=\mathcal{D}_{x}\left(\ln f_{b}\right) d x+\mathcal{D}_{y}\left(\ln f_{b}\right) d y-\mathcal{D}_{t}\left(\ln f_{b}\right) d t,
$$

so that our ansatz for $\omega$ is

$$
\omega=\Omega+\phi_{1} d x+\phi_{2} d y+\phi_{3} d t
$$

where $\phi_{k}$ are functions of the 2-jet of $u$.

Step (b): here we obtain only one non-trivial equation:

$$
f_{b b b}=2 \frac{f_{b b}^{2}}{f_{b}} .
$$

Step (c): eliminating the derivatives of $\phi_{k}$ with respect to the variables $u_{x x}, u_{x y}, u_{x t}, u_{y y}, u_{y t}$, we obtain a linear system for $\phi_{k}$ (which vanishes identically if $f_{b b}=0$, that is, if the original equation is of Monge-Ampère type). In the case $f_{b b} \neq 0$ this system gives an explicit formula for $\phi$ :

$$
\phi_{1}=0, \quad \phi_{2}=0, \quad \phi_{3}=-\frac{2}{3} f_{u_{t}}+\frac{8}{3} \hat{\mathcal{D}}_{t}\left(\log f_{b}\right)-\frac{4}{3} \hat{\mathcal{D}}_{t}\left(\log f_{b b}\right)
$$

thus leading to an explicit formula for the Weyl covector $\omega$. Here $\hat{\mathcal{D}}_{t}$ is the truncated total $t$-derivative (all differentiations are with respect to the 1 -jet variables only):

$$
\hat{\mathcal{D}}_{t}=\partial_{t}+u_{t} \partial_{u}+u_{x t} \partial_{u_{x}}+u_{y t} \partial_{u_{y}}+u_{t t} \partial_{u_{t}} .
$$


We will not continue with step (d): according to Sect. 2.1, it would lead to a conclusion that any equation (18) with EW property is point-equivalent to the BF equation $u_{t t}=\ln u_{x y}$.

Example 2. Let us consider equations of the form

$$
u_{t t}=f\left(x, y, t, u, u_{x}, u_{y}, u_{t}, u_{x x}, u_{y y}\right) .
$$

The characteristic conformal structure is ( $\left.\operatorname{set} a=u_{x x}, c=u_{y y}\right)$ :

$$
g=f_{c} d x^{2}+f_{a} d y^{2}-f_{a} f_{c} d t^{2} .
$$

We will assume that at least one of the second-order derivatives $f_{a a}, f_{a c}, f_{c c}$ is nonzero; otherwise the equation is of Monge-Ampère type (note also that $f_{a} \neq 0, f_{c} \neq 0$ if the equation is nondegenerate).

Step (a): calculation of $\Omega$ using formula (3) gives

$$
\Omega=2 \mathcal{D}_{x}\left(\ln f_{a}\right) d x+2 \mathcal{D}_{y}\left(\ln f_{c}\right) d y
$$

so that our ansatz for $\omega$ is

$$
\omega=\Omega+\phi_{1} d x+\phi_{2} d y+\phi_{3} d t
$$

where $\phi_{k}$ are functions of the 2-jet of $u$.

Step (b): here we obtain a system of PDEs for $f$ in the arguments $a, c$ :

$$
\begin{aligned}
f_{a a a} & =f_{a a}\left(\frac{f_{a c}}{f_{c}}+\frac{f_{a a}}{f_{a}}\right), \quad f_{a a c}=f_{a a}\left(\frac{f_{c c}}{f_{c}}+\frac{f_{a c}}{f_{a}}\right), \\
f_{a c c} & =f_{c c}\left(\frac{f_{c c}}{f_{c}}+\frac{f_{a c}}{f_{a}}\right), \quad f_{c c c}=f_{c c}\left(\frac{f_{c c}}{f_{c}}+\frac{f_{a c}}{f_{a}}\right), \quad f_{a a} f_{c c}=\left(f_{a c}\right)^{2} .
\end{aligned}
$$

These equations can be explicitly solved (see [24], Section 3.1).

Step (c): eliminating the derivatives of $\phi_{k}$ with respect to the variables $u_{x x}, u_{x y}, u_{x t}, u_{y y}, u_{y t}$, we obtain a linear system for $\phi_{k}$. The first few equations of this system are as follows:

$$
\begin{aligned}
f_{a} f_{c c} \phi_{1} & =f_{a c} \hat{\mathcal{D}}_{x}\left(f_{c}\right)-f_{c c} \hat{\mathcal{D}}_{x}\left(f_{a}\right), \quad f_{c} f_{a a} \phi_{2}=f_{a c} \hat{\mathcal{D}}_{y}\left(f_{a}\right)-f_{a a} \hat{\mathcal{D}}_{y}\left(f_{c}\right), \\
3 f_{a} f_{c} f_{c c} \phi_{2} & =4 f_{a} f_{c c} \hat{\mathcal{D}}_{y}\left(f_{c}\right)+4 f_{c} f_{c c} \hat{\mathcal{D}}_{y}\left(f_{a}\right)-4 f_{a} f_{c} \hat{\mathcal{D}}_{y}\left(f_{c c}\right)+2 f_{a} f_{c c} f_{u_{y}}, \\
3 f_{a} f_{c} f_{c c} \phi_{3} & =4 f_{a} f_{c c} \hat{\mathcal{D}}_{t}\left(f_{c}\right)+4 f_{c} f_{c c} \hat{\mathcal{D}}_{t}\left(f_{a}\right)-4 f_{a} f_{c} \hat{\mathcal{D}}_{t}\left(f_{c c}\right)-2 f_{a} f_{c c} f_{u_{t}}, \text { etc.; }
\end{aligned}
$$

here $\hat{\mathcal{D}}_{x}, \hat{\mathcal{D}}_{y}, \hat{\mathcal{D}}_{t}$ are the truncated total derivatives. In the non-Monge-Ampère case we can explicitly determine $\phi$ (and hence $\omega$ ). For instance, if $f_{c c} \neq 0$ then the first and the last two of the above equations give explicit values for $\phi_{1}, \phi_{2}, \phi_{3}$.

We will not continue with step (d), which would eventually lead to a conclusion that any equation (19) with EW property is contact-equivalent to an integrable Hirota type equation of the form $u_{t t}=f\left(u_{x x}, u_{y y}\right)$; see [24], Sect. 3.1, for a list of such equations. 


\section{Dispersionless Lax Pairs}

A background solution is the manifold $M=\mathbb{R}^{3}\left(x^{0}, x^{1}, x^{2}\right)$ or a domain thereof, equipped with a function $u$ solving (1). We encode it into the symbol $M_{u}$, which can be viewed as $\operatorname{graph}(u) \subset M \times \mathbb{R}$, as well as its lift into the jet-space inheriting the geometric structure. Of the latter we emphasise the characteristic variety, which is a projectivisation of the null cone of $[g]$ at every point. This bundle is four-dimensional, called the correspondence space $\hat{M}_{u}$.

Recall that a dispersionless Lax pair (dLp) can be identified with a rank 2 distribution $\hat{\Pi}$ in $\hat{M}_{u}$. The distribution $\hat{\Pi}$ depends on a finite jet of the solution $u$, and is Frobenius integrable modulo equation (1). The natural projection $\pi: \hat{M}_{u} \rightarrow M_{u}$ has projective fibre $\mathbb{P}^{1}$ with coordinate $\lambda$ called the spectral parameter; it parametrises null 2-planes $\Pi$ of the conformal structure $[g]$ on $M_{u}$.

It was shown in [5] that modulo equation (1) such Lax pair is unique, coisotropic with respect to the characteristic variety, and the lift $\Pi \rightarrow \hat{\Pi}$ has the projective property. In Lemma 4 of [5] it was proved that the Weyl covector $\omega$ uniquely determines the lift (see also Lemma 5 of [5]); however, no explicit formula for the lift was provided. This is what we do below in the proof of Theorem 2.

\subsection{Proof of Theorem 2}

Let $X, Y$ be $\lambda$-dependent vector fields generating $\Pi$, and let

$$
\hat{X}=X+m \partial_{\lambda}, \quad \hat{Y}=Y+n \partial_{\lambda}
$$

be their lifts to $\hat{\Pi}$. A section $\lambda=\lambda(\boldsymbol{x})$ is foliated by a one-parametric family of integral surfaces of $\hat{\Pi}$ iff

$$
\hat{X}(\lambda-\lambda(\boldsymbol{x}))=m-X(\lambda(\boldsymbol{x}))=0, \quad \hat{Y}(\lambda-\lambda(\boldsymbol{x}))=n-Y(\lambda(\boldsymbol{x}))=0 .
$$

This gives

$$
m=X(\lambda(\boldsymbol{x})), \quad n=Y(\lambda(\boldsymbol{x})),
$$

and it remains to show that all first-order derivatives of $\lambda$ on the right-hand sides of (20) can be eliminated. Let $\theta \in \Pi^{\perp}$ be a ( $\lambda$-dependent) annihilator of the 2-plane congruence $\Pi$. The condition that the Weyl connection $\mathbb{D}$ preserves the field of null cones is

$$
\mathbb{D}_{X} \theta \wedge \theta=0, \quad \mathbb{D}_{Y} \theta \wedge \theta=0,
$$

where we substitute $\lambda=\lambda(\boldsymbol{x})$ prior to differentiation. This condition gives precisely two linearly independent equations on the 1 -jet of $\lambda(\boldsymbol{x})$, and these imply that all derivatives of $\lambda$ on the right-hand sides of (20) cancel out, leading to the required formulae for $m$ and $n$.

Given $g, \omega$ and following the above scheme, let us derive an explicit formula for dLp.

First of all, we choose a (nonholonomic) null coframe $\theta^{0}, \theta^{1}, \theta^{2}$ such that

$$
g=4 \theta^{0} \theta^{2}-\left(\theta^{1}\right)^{2} .
$$


Let $V_{0}, V_{1}, V_{2}$ be the dual frame, and let $c_{i j}^{k}$ be the structure functions defined by the expansions

$$
\left[V_{i}, V_{j}\right]=c_{i j}^{k} V_{k} \quad \Leftrightarrow \quad d \theta^{k}=-\sum_{i<j} c_{i j}^{k} \theta^{i} \wedge \theta^{j} .
$$

The 2-plane congruence is $\Pi=\left\langle X=V_{0}+\lambda V_{1}, Y=V_{1}+\lambda V_{2}\right\rangle$ and $\theta(\lambda)=$ $\theta_{2}-\lambda \theta_{1}+\lambda^{2} \theta_{0}$. Representing the Weyl covector $\omega$ in the form $\omega=\omega_{i} \theta^{i}$ we compute the Weyl connection $\mathbb{D}$ :

$$
\begin{aligned}
\mathbb{D} \theta^{0}= & \left(c_{02}^{2}+4 \omega_{0}\right) \theta^{0} \otimes \theta^{0}+\left(\frac{1}{2} c_{12}^{2}+2 \omega_{1}-\frac{1}{2} c_{01}^{0}-\frac{1}{4} c_{02}^{1}\right) \theta^{0} \otimes \theta^{1} \\
& +\left(\frac{1}{2} c_{01}^{0}+\frac{1}{2} c_{12}^{2}+2 \omega_{1}-\frac{1}{4} c_{02}^{1}\right) \theta^{1} \otimes \theta^{0} \\
& +\left(\frac{1}{4} \omega_{2}-\frac{1}{2} c_{12}^{1}\right) \theta^{1} \otimes \theta^{1}+c_{02}^{0} \theta^{2} \otimes \theta^{0}+c_{12}^{0} \theta^{2} \otimes \theta^{1}, \\
\mathbb{D} \theta^{1}= & -2 c_{01}^{2} \theta^{0} \otimes \theta^{0}+\frac{1}{2} \omega_{0} \theta^{0} \otimes \theta^{1}+\left(c_{12}^{2}-c_{01}^{0}+4 \omega_{1}-\frac{1}{2} c_{02}^{1}\right) \theta^{0} \otimes \theta^{2} \\
& +\left(c_{01}^{1}+\frac{1}{2} \omega_{0}\right) \theta^{1} \otimes \theta^{0}+\frac{1}{2} \omega_{1} \theta^{1} \otimes \theta^{1}+\left(\frac{1}{2} \omega_{2}-c_{12}^{1}\right) \theta^{1} \otimes \theta^{2} \\
& +\left(c_{12}^{2}+4 \omega_{1}-c_{01}^{0}+\frac{1}{2} c_{02}^{1}\right) \theta^{2} \otimes \theta^{0}+\frac{1}{2} \omega_{2} \theta^{2} \otimes \theta^{1}+2 c_{12}^{0} \theta^{2} \otimes \theta^{2}, \\
\mathbb{D} \theta^{2}= & -c_{01}^{2} \theta^{0} \otimes \theta^{1}-c_{02}^{2} \theta^{0} \otimes \theta^{2}+\left(\frac{1}{2} c_{01}^{1}+\frac{1}{4} \omega_{0}\right) \theta^{1} \otimes \theta^{1} \\
& +\left(\frac{1}{4} c_{02}^{1}+2 \omega_{1}-\frac{1}{2} c_{12}^{2}-\frac{1}{2} c_{01}^{0}\right) \theta^{1} \otimes \theta^{2} \\
& +\left(\frac{1}{4} c_{02}^{1}+2 \omega_{1}+\frac{1}{2} c_{12}^{2}-\frac{1}{2} c_{01}^{0}\right) \theta^{2} \otimes \theta^{1}+\left(4 \omega_{2}-c_{02}^{0}\right) \theta^{2} \otimes \theta^{2} .
\end{aligned}
$$

Our convention is $\mathbb{D} \theta^{k}=-\Gamma_{i j}^{k} \theta^{i} \otimes \theta^{j} \Leftrightarrow \mathbb{D}_{V_{i}} \theta^{k}=-\Gamma_{i j}^{k} \theta^{j}$. The torsion-free condition is equivalent to alt $\left(\mathbb{D} \theta^{k}\right)=\frac{1}{2} d \theta^{k}$, and we also have $\mathbb{D} g=\omega \otimes g$. Finally, using (21) we compute the dLp to be

$$
\hat{\Pi}=\left\langle\hat{X}=V_{0}+\lambda V_{1}+m \partial_{\lambda}, \quad \hat{Y}=V_{1}+\lambda V_{2}+n \partial_{\lambda}\right\rangle,
$$

with

$$
\begin{aligned}
m & =\left(\frac{1}{2} c_{12}^{1}-\frac{1}{4} \omega_{2}\right) \lambda^{3}+\left(\frac{1}{2} c_{02}^{1}-c_{12}^{2}-\frac{1}{2} \omega_{1}\right) \lambda^{2}+\left(\frac{1}{2} c_{01}^{1}-c_{02}^{2}-\frac{1}{4} \omega_{0}\right) \lambda-c_{01}^{2}, \\
n & =-c_{12}^{0} \lambda^{3}+\left(\frac{1}{2} c_{12}^{1}-c_{02}^{0}+\frac{1}{4} \omega_{2}\right) \lambda^{2}+\left(\frac{1}{2} c_{02}^{1}-c_{01}^{0}+\frac{1}{2} \omega_{1}\right) \lambda+\left(\frac{1}{2} c_{01}^{1}+\frac{1}{4} \omega_{0}\right) .
\end{aligned}
$$

Let us also note, following [5], $\$ 4.3$, that the lift of $W=V_{0}+2 \lambda V_{1}+\lambda^{2} V_{2}$ does not depend on the Weyl connection and equals $\hat{W}=W+\sigma \partial_{\lambda}$ with $\sigma=m+n \lambda=-c_{12}^{0} \lambda^{4}+\left(c_{12}^{1}-c_{02}^{0}\right) \lambda^{3}-\left(c_{01}^{0}-c_{02}^{1}+c_{12}^{2}\right) \lambda^{2}+\left(c_{01}^{1}-c_{02}^{2}\right) \lambda-c_{01}^{2}$.

This is related to the fact that $W$ is null and is therefore independent of the choice of $\omega$. The lift of other vectors from $\Pi$ does depend on $\omega$.

As the covector $\omega$ is algebraically determined by the equation, the Lax pair is also explicitly determined, thus finishing the proof of Theorem 2 .

\subsection{Examples of Computations}

Below we discuss several examples illustrating the calculations described in the proof.

Example 1. For the dispersionless Kadomtsev-Petviashvili (dKP) equation, $u_{x t}=\left(u u_{x}\right)_{x}+u_{y y}$, the conformal structure is

$$
g=4 d x d t-d y^{2}+4 u d t^{2}
$$


and the Weyl covector is $\omega=-4 u_{x} d t$. The corresponding Weyl connection $\mathbb{D}$ is given by the following nontrivial relations:

$$
\begin{aligned}
& \mathbb{D}_{\partial_{x}} \partial_{t}=\mathbb{D}_{\partial_{t}} \partial_{x}=\mathbb{D}_{\partial_{y}} \partial_{y}=u_{x} \partial_{x}, \quad \mathbb{D}_{\partial_{y}} \partial_{t}=\mathbb{D}_{\partial_{t}} \partial_{y}=u_{y} \partial_{t}+2 u_{x} \partial_{y}, \\
& \mathbb{D}_{\partial_{t}} \partial_{t}=\left(u_{t}-2 u u_{x}\right) \partial_{x}+2 u_{y} \partial_{y}+3 u_{x} \partial_{t} .
\end{aligned}
$$

We have $\theta(\lambda)=d x+\lambda d y+\left(\lambda^{2}+u\right) d t$ and

$$
\Pi=\operatorname{Ann}(\theta)=\left\langle X=\partial_{y}-\lambda \partial_{x}, Y=\partial_{t}-\left(\lambda^{2}+u\right) \partial_{x}\right\rangle .
$$

Condition (21) gives

$$
\lambda_{t}=\lambda^{2} \lambda_{x}+\lambda u_{x}+u \lambda_{x}+u_{y}, \quad \lambda_{y}=\lambda \lambda_{x}+u_{x},
$$

leading to the familiar dLp for the dKP equation: $\hat{X}=X+m \partial_{\lambda}, \hat{Y}=Y+n \partial_{\lambda}$ with

$$
m=\lambda_{y}-\lambda \lambda_{x}=u_{x}, \quad n=\lambda_{t}-\left(\lambda^{2}+u\right) \lambda_{x}=\lambda u_{x}+u_{y} .
$$

Example 2. For the dispersionless lattice equations (4) we have

$$
\theta_{0}=f_{u_{t t}} d y, \theta_{1}=d t, \theta_{2}=d x,
$$

so that $c_{01}^{0}=\mathcal{D}_{t} \ln f_{u_{t t}}, c_{02}^{0}=\mathcal{D}_{x} \ln f_{u_{t t}}$ and $\omega_{1}=\omega\left(\partial_{t}\right)$ are the only nonzero entries in the above formulae, giving

$$
m=-\frac{1}{2} \omega_{1} \lambda^{2}, \quad n=-\mathcal{D}_{x} \ln f_{u_{t t}} \lambda^{2}+\left(\frac{1}{2} \omega_{1}-\mathcal{D}_{t} \ln f_{u_{t t}}\right) \lambda .
$$

This reproduces the Lax pair from Sect. 2.1.

Example 3. For the generalised Dunajski-Tod equations (7) it is convenient to change the representative of the conformal class as follows:

$g=4 d x d y-\alpha^{2}$, where $\alpha=\frac{1}{\sqrt{-f}}\left(\left(u_{x t}-u_{x}\right) d x+\left(u_{y t}+u_{y}\right) d y+\left(u_{t t}-u\right) d t\right)$.

Then $\omega$ is changed to the new Weyl covector

$$
\omega_{\text {new }}=\omega-d \ln |f|=\omega_{0} \theta^{0}+\omega_{1} \theta^{1}+\omega_{2} \theta^{2}
$$

where $\theta^{0}=d x, \theta^{1}=\alpha, \theta^{2}=d y$ is a null coframe. The dual frame is

$$
V_{0}=\partial_{x}-\frac{u_{x t}-u_{x}}{u_{t t}-u} \partial_{t}, \quad V_{1}=\frac{\sqrt{-f}}{u_{t t}-u} \partial_{t}, \quad V_{2}=\partial_{y}-\frac{u_{y t}+u_{y}}{u_{t t}-u} \partial_{t} .
$$

The coefficients $\omega_{i}=\omega_{\text {new }}\left(V_{i}\right)$ are given by

$$
\begin{aligned}
& \omega_{0}=2(1+R) \frac{u_{x t}-u_{x}}{u_{t t}-u}-\mathcal{D}_{x} \ln |f|, \quad \omega_{1}=\frac{2 R \sqrt{-f}}{u_{t t}-u}, \\
& \omega_{2}=2(1+R) \frac{u_{y t}+u_{y}}{u_{t t}-u}-\mathcal{D}_{y} \ln |f| .
\end{aligned}
$$

The only nonzero structure functions are

$$
c_{01}^{1}=d \alpha\left(V_{1}, V_{0}\right), c_{02}^{1}=d \alpha\left(V_{2}, V_{0}\right), c_{12}^{1}=d \alpha\left(V_{2}, V_{1}\right),
$$

thus giving the Lax pair

$$
\hat{X}=V_{0}+\lambda V_{1}+m \partial_{\lambda}, \quad \hat{Y}=V_{1}+\lambda V_{2}+n \partial_{\lambda},
$$


where

$$
\begin{aligned}
m & =\left(\frac{1}{2} c_{12}^{1}-\frac{1}{4} \omega_{2}\right) \lambda^{3}+\left(\frac{1}{2} c_{02}^{1}-\frac{1}{2} \omega_{1}\right) \lambda^{2}+\left(\frac{1}{2} c_{01}^{1}-\frac{1}{4} \omega_{0}\right) \lambda, \\
n & =\left(\frac{1}{2} c_{12}^{1}+\frac{1}{4} \omega_{2}\right) \lambda^{2}+\left(\frac{1}{2} c_{02}^{1}+\frac{1}{2} \omega_{1}\right) \lambda+\left(\frac{1}{2} c_{01}^{1}+\frac{1}{4} \omega_{0}\right) .
\end{aligned}
$$

\section{Rigidity Conjecture}

Consider equation (1) which satisfies EW property. As explained in Sect. 3, step (b), freezing in (1) the 1-jet of $u$ we obtain an integrable Hirota-type equation. It was demonstrated in [24] that the parameter space of integrable Hirota-type equations is 21-dimensional, supplied with a locally free action of the 21-dimensional equivalence group $\mathbf{S p}(6, \mathbb{R})$. This implies the existence of a universal Hirota master-equation generating an open $\mathbf{S p}(6, \mathbb{R})$-orbit in the 21-dimensional parameter space. It was shown in [21] that integrability of Hirota-type equations is equivalent to the EW property, with the Weyl covector $\omega$ given by formula (3). Finally, it was proved in [10] that the Hirota master-equation, which is a highly transcendental object, coincides with the equation of the genus three hyperelliptic divisor. We will say that PDE (1) is generic if a Hirota-type equation obtained by freezing a generic 1-jet of $u$ belongs to the open orbit.

Conjecture. A generic second-order PDE (1) satisfying EW property is equivalent to the Hirota master-equation via a suitable contact transformation. In other words, for a generic PDE satisfying EW property, all dependence on the 1-jets is not essential, and can be eliminated by a change of variables. In fact, examples suggest that the 'genericity' assumption can be weakened, leading to the following stronger conjecture: if a second-order PDE (which is not Monge-Ampère) satisfies EW property, then it is equivalent to a Hirotatype equation via a contact transformation.

An illustration of the rigidity phenomenon is given by the classification result of Sect. 2.1, where it was shown that any equation of type (4) satisfying $\mathrm{EW}$ property is reducible to the BF equation $u_{x y}=e^{u_{z z}}$. For MongeAmpère equations the conjecture is certainly not true: there exist contact non-equivalent examples satisfying EW property (see Sects. 2.2 and 2.3, and Concluding Remarks for a discussion).

Below we prove two rigidity-type results, which motivate the above conjecture and demonstrate a technique that may be utilised in its proof in full generality. The main tool is the existence of an open orbit in the solution space of some differential equations with respect to their point symmetry groups.

\subsection{Rigidity Result 1}

Let us consider Lagrangians of the form

$$
\int u_{x} u_{y} \varphi\left(u_{t}\right) d x d y d t
$$

It was shown in [25] that the requirement of integrability (EW property) of the corresponding second-order Euler-Lagrange equation implies that the function 
$\varphi(z)$ satisfies a fourth-order ODE

$$
\varphi^{\prime \prime \prime \prime}\left(\varphi^{2} \varphi^{\prime \prime}-2 \varphi \varphi^{\prime 2}\right)-9 \varphi^{\prime 2} \varphi^{\prime \prime 2}+2 \varphi \varphi^{\prime} \varphi^{\prime \prime} \varphi^{\prime \prime \prime}+8 \varphi^{\prime 3} \varphi^{\prime \prime \prime}-\varphi^{2} \varphi^{\prime \prime \prime 2}=0,
$$

whose general solution is a modular form of weight one and level three known as the Eisenstein series $E_{1,3}(z)$.

Proposition 1. Every Lagrangian of the form

$$
\int u_{x} u_{y} f\left(t, u, u_{t}\right) d x d y d t
$$

whose Euler-Lagrange equation satisfies EW property, is equivalent to its undeformed version (22) via a change of variables. In other words, Lagrangian (22) is rigid within the class (24).

Proof. The Euler-Lagrange equation for Lagrangian (24) is

$u_{x} u_{y} f_{u_{t}, u_{t}} u_{t t}+2 f u_{x y}+2 f_{u_{t}} u_{y} u_{x t}+2 f_{u_{t}} u_{x} u_{y t}+u_{x} u_{y}\left(f_{u}+f_{u_{t}, t}+u_{t} f_{u_{t}, u}\right)=0$.

The corresponding characteristic conformal structure is

$$
g=-\left(f_{u_{t}} u_{x} d x+f_{u_{t}} u_{y} d y-f d t\right)^{2}+2 u_{x} u_{y}\left(2 f_{u_{t}}^{2}-f f_{u_{t}, u_{t}}\right) .
$$

Looking for $\omega$ in form (15) and substituting into the Einstein-Weyl conditions we obtain

$$
\phi_{1}=-u_{x} \frac{f_{u_{t}}}{f} Z, \quad \phi_{2}=-u_{y} \frac{f_{u_{t}}}{f} Z, \quad \phi_{3}=Z,
$$

where

$$
Z=\frac{6 f_{u} f_{u_{t}}-2 f_{u_{t}} f_{u_{t}, t}+2\left(f_{t}+f_{u} u_{t}\right) f_{u_{t}, u_{t}}-2\left(2 f+f_{u_{t}} u_{t}\right) f_{u_{t}, u}}{f f_{u_{t}, u_{t}}-2 f_{u_{t}}^{2}},
$$

(confirming that $\omega$ can be expressed in terms of the equation). Furthermore, we obtain four differential constraints for the function $f\left(t, u, u_{t}\right)$ : one of them coincides with the ODE (23) in the variable $u_{t}=z$, while the other three are more complicated. Utilising GL(2)-invariance of ODE (23) [25], we look for a general solution in the form

$$
f\left(t, u, u_{t}\right)=\frac{q}{\gamma u_{t}+\delta} \varphi\left(\frac{\alpha u_{t}+\beta}{\gamma u_{t}+\delta}\right),
$$

where $\varphi(z)$ is a generic solution of (23), and $\alpha, \beta, \gamma, \delta, q$ should be considered as functions of the remaining arguments $t, u$. Under this ansatz, the ODE (23) (in the variable $u_{t}=z$ ) will be automatically satisfied. Direct analysis of the remaining constraints reveals that there exists a common factor $p$ such that $(p \alpha)_{t}=(p \beta)_{u}$ and $(p \gamma)_{t}=(p \delta)_{u}$. Introducing the potentials one can set

$$
f\left(t, u, u_{t}\right)=\frac{q}{h_{u} u_{t}+h_{t}} \varphi\left(\frac{g_{u} u_{t}+g_{t}}{h_{u} u_{t}+h_{t}}\right),
$$

where $q$ can be reconstructed uniquely up to a constant factor, leading to the following final answer:

$$
f\left(t, u, u_{t}\right)=\frac{\left(g_{u} h_{t}-h_{u} g_{t}\right)^{2}}{h_{u} u_{t}+h_{t}} \varphi\left(\frac{g_{u} u_{t}+g_{t}}{h_{u} u_{t}+h_{t}}\right)
$$


here $h(t, u)$ and $g(t, u)$ are two arbitrary functions. With any $f\left(t, u, u_{t}\right)$ given by (26), equation (25) possesses EW property. It is a common phenomenon that arbitrary functions occurring in the coefficients of integrable systems can be eliminated by a change of variables. This is exactly the case in our example: introducing the point transformation

$$
X=x, \quad Y=y, \quad T=h(t, u), \quad U=g(t, u),
$$

one can show that the densities

$$
U_{X} U_{Y} \varphi\left(U_{T}\right) d X \wedge d Y \wedge d T \text { and } u_{x} u_{y} f\left(t, u, u_{t}\right) d x \wedge d y \wedge d t
$$

transform into each other, thus establishing triviality of deformation (24).

\subsection{Rigidity Result 2}

Equations of the form

$$
u_{t t}=\frac{u_{x y}}{u_{x t}}+\frac{1}{6} \varphi\left(u_{x x}\right) u_{x t}^{2}
$$

have appeared in the classification of integrable hydrodynamic chains [31], where it was shown that $\varphi$ must satisfy the Chazy equation [8] (set $\left.u_{x x}=a\right)$ :

$$
\varphi_{a a a}+2 \varphi \varphi_{a a}-3 \varphi_{a}^{2}=0 .
$$

Proposition 2. Every equation of the form

$$
u_{t t}=\frac{u_{x y}}{u_{x t}}+\frac{1}{6} f\left(x, u, u_{x}, u_{x x}\right) u_{x t}^{2},
$$

which satisfies EW property, is equivalent to its undeformed version (27) via a suitable contact transformation. In other words, equation (27) is rigid within the class (29).

Proof. The corresponding characteristic conformal structure is

$$
\begin{aligned}
g= & u_{x t}^{3} d x d y-\left(\frac{1}{6} f_{a} u_{x t}^{6}+\frac{1}{4}\left(u_{x y}-\frac{1}{3} f u_{x t}^{3}\right)^{2}\right) d y^{2}-\frac{1}{2} u_{x t}\left(u_{x y}-\frac{1}{3} f u_{x t}^{3}\right) d y d t \\
& -\frac{1}{4} u_{x t}^{2} d t^{2} .
\end{aligned}
$$

Looking for $\omega$ in form (15) and substituting into the Einstein-Weyl conditions we obtain

$$
\phi_{1}=\phi_{3}=0, \quad \phi_{2}=-\frac{2}{3} u_{x t}^{3} f_{u_{x}}
$$

(once again confirming that $\omega$ can be expressed in terms of the equation). Furthermore, we obtain the condition $f_{u}=0$, as well as four differential constraints for the function $f\left(x, u_{x}, u_{x x}\right)$ : one of them coincides with the Chazy equation (28) in the variable $u_{x x}=a$, while the other three are more complicated. Utilising SL(2)-invariance of the Chazy equation [9], we look for a general solution in the form

$$
f\left(x, u_{x}, u_{x x}\right)=\frac{1}{\left(\gamma u_{x x}+\delta\right)^{2}} \varphi\left(\frac{\alpha u_{x x}+\beta}{\gamma u_{x x}+\delta}\right)+\frac{6 \gamma}{\gamma u_{x x}+\delta}, \quad \alpha \delta-\beta \gamma=1,
$$


where $\varphi(a)$ is a generic solution of (28), and $\alpha, \beta, \gamma, \delta$ should be considered as functions of the remaining arguments $x, u_{x}$. Under this ansatz, the Chazy equation (28) will be automatically satisfied. Direct analysis of the remaining constraints reveals that, analogously to the previous example, there exist potentials $g\left(x, u_{x}\right)$ and $h\left(x, u_{x}\right)$ such that $\alpha=g_{u_{x}}, \beta=g_{x}, \gamma=h_{u_{x}}, \delta=h_{x}$. This leads to the following final answer:

$$
f\left(x, u_{x}, u_{x x}\right)=\frac{1}{\left(h_{u_{x}} u_{x x}+h_{x}\right)^{2}} \varphi\left(\frac{g_{u_{x}} u_{x x}+g_{x}}{h_{u_{x}} u_{x x}+h_{x}}\right)+\frac{6 h_{u_{x}}}{h_{u_{x}} u_{x x}+h_{x}} ;
$$

here $g\left(x, u_{x}\right)$ and $h\left(x, u_{x}\right)$ are two functions which satisfy a single constraint $g_{u_{x}} h_{x}-h_{u_{x}} g_{x}=1$ (corollary of $\alpha \delta-\beta \gamma=1$ ). With any $f\left(x, u_{x}, u_{x x}\right)$ given by (30), equation (29) possesses EW property. To eliminate arbitrary functions let us use the potential substitution $v=u_{x}$ which reduces equation (27) to quasilinear form

$$
v_{t t}=\left(\frac{v_{y}}{v_{t}}+\frac{1}{6} f\left(x, v, v_{x}\right) v_{t}^{2}\right)_{x} .
$$

Equation (31) can be equivalently represented as the condition of closedness of the 2 -form

$$
v_{t} d x \wedge d y+\left(\frac{v_{y}}{v_{t}}+\frac{1}{6} f\left(x, v, v_{x}\right) v_{t}^{2}\right) d t \wedge d y .
$$

Introducing the point transformation

$$
Y=y, \quad T=t, \quad X=h(x, v), \quad V=g(x, v),
$$

one can verify the identity

$$
\begin{array}{rl}
V_{T} & d X \wedge d Y+\left(\frac{V_{Y}}{V_{T}}+\frac{1}{6} \varphi\left(V_{X}\right) V_{T}^{2}\right) d T \wedge d Y \\
= & v_{t} d x \wedge d y+\left(\frac{v_{y}}{v_{t}}+\frac{1}{6} f\left(x, v, v_{x}\right) v_{t}^{2}\right) d t \wedge d y,
\end{array}
$$

thus demonstrating triviality of deformation (29) at quasilinear level (31). The composition of point transformation (32) with the potential substitution $v=u_{x}$ gives a contact transformation

$$
\begin{aligned}
X & =h\left(x, u_{x}\right), \quad Y=y, \quad T=t, \quad U=u+q\left(x, u_{x}\right), \\
U_{X} & =g\left(x, u_{x}\right), \quad U_{Y}=u_{y}, \quad U_{T}=u_{t},
\end{aligned}
$$

where $q\left(x, u_{x}\right)$ is defined by the equations

$$
q_{u_{x}}=g h_{u_{x}}, \quad q_{x}=g h_{x}-u_{x}
$$

(which are compatible by virtue of the relation $g_{u_{x}} h_{x}-h_{u_{x}} g_{x}=1$ ). Contact transformation (33) takes equation (29) with $f\left(x, u_{x}, u_{x x}\right)$ given by (30) to the undeformed equation

$$
U_{T T}=\frac{U_{X Y}}{U_{X T}}+\frac{1}{6} \varphi\left(U_{X X}\right) U_{X T}^{2}
$$

thus establishing the required triviality. 


\subsection{General Rigidity Conjecture}

The above arguments can be extended to the general case as follows. Let $U$ be the Hessian matrix of a function $u$, and let $F(U)=0$ be the Hirota master-equation. We will exploit two facts (we change to complex coefficients for classification reasons):

- Generic integrable Hirota-type equations belong to the same open $\mathbf{S p}(6, \mathbb{C})$ orbit, see [24];

- Freezing 1-jet of $u$ in equation (1) with EW property yields an integrable Hirota-type PDE, see $\S 3.2(\mathrm{~b})$.

Thus, a generic second-order PDE with EW property can be represented in the form

$$
F\left((A U+B)(C U+D)^{-1}\right)=0
$$

where $A, B, C, D$ are $3 \times 3$ matrices depending on 1 -jet variables $x^{i}, u, u_{i}$, such that the $6 \times 6$ matrix

$$
\left(\begin{array}{ll}
A & B \\
C & D
\end{array}\right)
$$

belongs to $\operatorname{Sp}(6, \mathbb{C})$. Under the substitution $(34)$ part of the Einstein-Weyl conditions will be satisfied identically, leaving a (complicated!) system of differential constraints for $A, B, C, D$ in the 1 -jet variables. It remains to prove that these constraints are equivalent to the existence of a contact transformation taking PDE (34) into Hirota form $F(U)=0$. The complexity of the resulting differential constraints is a formidable obstacle in this programme.

\section{Concluding remarks}

1. In this paper, we have studied second-order PDEs in 3D whose characteristic conformal structure is Einstein-Weyl on every solution. A special subclass thereof are PDEs whose characteristic conformal structure is flat on every solution (that is, has zero Cotton tensor). We conjecture that any such PDE is contact equivalent to $\triangle u=s$ where $\triangle$ denotes the Laplace operator of a constant-coefficient metric and $s$ is some function depending on the 1-jet of $u$. In other words, if the characteristic conformal structure is flat on every solution, the principal symbol can be reduced to constant-coefficient form for all solutions simultaneously, via a suitable contact transformation. This result should be true in any dimension higher than two (in two dimensions this is clearly false).

2. We have demonstrated the existence of a formula for the Weyl covector $\omega$ for PDEs (1) that satisfy EW property and do not belong to the Monge-Ampère class. This formula came from the terms in the EinsteinWeyl equations that are linear in the third-order derivatives of $u$. We expect that analogous formula can be constructed for all second-order PDEs whose characteristic conformal structure $[g]$ is not flat on generic solution (the required formula should follow from the overdetermined system formed by the terms depending on 2-jet of $u$ ). More generally, 
we conjecture that such a formula exists for any PDE system whose characteristic variety is a nondegenerate quadric, yet the corresponding conformal structure $[g]$ is not flat on generic solution. This would include the Manakov-Santini system, thus agreeing with the results established in $[5]$.

3. It was observed in [21] that second-order PDEs in 3D that are integrable by the method of hydrodynamic reductions [22] must necessarily have EW property. Since EW property (unlike hydrodynamic integrability) is manifestly contact-invariant, it is tempting to adopt it as a contactinvariant approach to dispersionless integrability. This would have a serious drawback: it is unknown at present how to solve such equations, indeed, multiphase solutions coming from the method of hydrodynamic reductions may not be available. On the other hand, we conjectured that non-Monge-Ampère second-order PDEs with EW property are in a sense 'rigid': they can be reduced to dispersionless Hirota form $F\left(u_{x^{i} x^{j}}\right)=0$ via a suitable contact transformation. More generally, we expect that Monge-Ampère equations with Einstein-Weyl characteristic conformal structure (which is not flat on every solution) can be transformed, via a suitable Bäcklund transformation, into a translationally invariant form to which the method of hydrodynamic reductions would already apply. Note that in the latter case contact transformations may not be sufficient.

As an illustrating example consider the following translationally noninvariant integrable deformation of the Veronese web equation,

$$
\left(x^{1}-x^{2}\right) u_{x^{3}} u_{x^{1} x^{2}}+\left(x^{2}-x^{3}\right) u_{x^{1}} u_{x^{2} x^{3}}+\left(x^{3}-x^{1}\right) u_{x^{2}} u_{x^{1} x^{3}}=0 .
$$

We refer to [29], Theorem 8.1 for the corresponding Einstein-Weyl structure; see also [30]. Introducing the one-form

$$
\begin{aligned}
\theta(\lambda)= & \left(\lambda-x^{2}\right)\left(\lambda-x^{3}\right) u_{x^{1}} d x^{1}+\left(\lambda-x^{1}\right)\left(\lambda-x^{3}\right) u_{x^{2}} d x^{2} \\
& +\left(\lambda-x^{1}\right)\left(\lambda-x^{2}\right) u_{x^{3}} d x^{3},
\end{aligned}
$$

(here $\lambda$ is a constant parameter), one can represent equation (35) in compact form $d \theta(\lambda) \wedge \theta(\lambda)=0$. Equation (35) is not contact-equivalent to any translationally invariant equation. Indeed, if it was, it would possess a threedimensional commutative subalgebra of contact symmetries (corresponding to translations in the independent variables). However, the contact symmetry algebra of equation (35) is generated by vector fields

$$
f(u) \partial_{u}, \quad \partial_{x^{1}}+\partial_{x^{2}}+\partial_{x^{3}}, \quad x^{1} \partial_{x^{1}}+x^{2} \partial_{x^{2}}+x^{3} \partial_{x^{3}},
$$

and one can easily see that this algebra does not contain any three-dimensional commutative subalgebra. Nonetheless, equation (35) is Bäcklund-related to the translationally invariant Veronese web equation [34],

$$
\left(a_{1}-a_{2}\right) u_{y^{3}} u_{y^{1} y^{2}}+\left(a_{2}-a_{3}\right) u_{y^{1}} u_{y^{2} y^{3}}+\left(a_{3}-a_{1}\right) u_{y^{2}} u_{y^{1} y^{3}}=0
$$

$a_{i}=$ const, which can be represented as $d \Theta(\lambda) \wedge \Theta(\lambda)=0$ where

$$
\begin{aligned}
\Theta(\lambda)= & \left(\lambda-a_{2}\right)\left(\lambda-a_{3}\right) u_{y^{1}} d y^{1}+\left(\lambda-a_{1}\right)\left(\lambda-a_{3}\right) u_{y^{2}} d y^{2} \\
& +\left(\lambda-a_{1}\right)\left(\lambda-a_{2}\right) u_{y^{3}} d y^{3} .
\end{aligned}
$$


A Bäcklund transformation between equations (35) and (36) can be represented in the form

$$
\Theta(\lambda)=\theta(\lambda)
$$

This is a nonlocal change of the independent variables $x \rightarrow y$, note that the dependent variable $u$ remains unchanged. Setting in (37) successively $\lambda=a_{i}$ we obtain

$u_{y^{1}} d y^{1}=\frac{\theta\left(a_{1}\right)}{\left(a_{1}-a_{2}\right)\left(a_{1}-a_{3}\right)}, \quad u_{y^{2}} d y^{2}=\frac{\theta\left(a_{2}\right)}{\left(a_{2}-a_{1}\right)\left(a_{2}-a_{3}\right)}, \quad u_{y^{3}} d y^{3}=\frac{\theta\left(a_{3}\right)}{\left(a_{3}-a_{1}\right)\left(a_{3}-a_{2}\right)}$.

These relations specify the new independent variables $y^{i}$ uniquely modulo transformations $y^{i} \rightarrow \varphi^{i}\left(y^{i}\right)$, which are point symmetries of equation (36). Bäcklund transformation (38) can be viewed as a $3 \mathrm{D}$ version of reciprocal transformations that are well-studied in $2 \mathrm{D}$.

\section{Acknowledgements}

We thank D. Calderbank, B. Doubrov, M. Dunajski and M. Pavlov for clarifying discussions. We also thank the referee for useful comments. The research of EVF was supported by a grant from the Russian Science Foundation No. 21-11-00006, https://rscf.ru/project/21-11-00006/. The work of BSK was partially supported by the project Pure Mathematics in Norway, funded by Trond Mohn Foundation and Troms $\varnothing$ Research Foundation. The research of VSN was supported by the EPSRC Grant EP/V050451/1.

Open Access. This article is licensed under a Creative Commons Attribution 4.0 International License, which permits use, sharing, adaptation, distribution and reproduction in any medium or format, as long as you give appropriate credit to the original author(s) and the source, provide a link to the Creative Commons licence, and indicate if changes were made. The images or other third party material in this article are included in the article's Creative Commons licence, unless indicated otherwise in a credit line to the material. If material is not included in the article's Creative Commons licence and your intended use is not permitted by statutory regulation or exceeds the permitted use, you will need to obtain permission directly from the copyright holder. To view a copy of this licence, visit http://creativecommons. org/licenses/by/4.0/.

Publisher's Note Springer Nature remains neutral with regard to jurisdictional claims in published maps and institutional affiliations.

\section{References}

[1] Bogdanov, L.V.: Non-Hamiltonian generalizations of the dispersionless 2DTL hierarchy. J. Phys. A 43(43), 434008 (2010)

[2] Boillat, G..: Sur l'équation générale de Monge-Ampère á plusieurs variables. C. R. Acad. Sci. Paris Sér. I Math. 313(11), 805-808 (1991)

[3] Boyer, C.P., Finley, J.D.: Killing vectors in self-dual Euclidean Einstein spaces. J. Math. Phys. 23, 1126-1130 (1982) 
[4] Calderbank, D.M.J.: Integrable background geometries. SIGMA 10, 034 (2014)

[5] Calderbank, D.M.J., Kruglikov, B.: Integrability via geometry: dispersionless differential equations in three and four dimensions. Commun. Math. Phys. 382, 1811-1841 (2021)

[6] Cartan, E.: Sur une classe d'espaces de Weyl. Ann. Sci. École Norm. Sup. 3(60), 1-16 (1943)

[7] Cartan, E.: The geometry of differential equations of third order. Revista Mat. Hisp.-Am. 4, 3-33 (1941)

[8] Chazy, J..: Sur les équations différentiellles dont l'intégrale générale possède un coupure essentielle mobile. C.R. Acad. Sci. Paris 150, 456-458 (1910)

[9] Clarkson, P.A., Olver, P.J.: Symmetry and the Chazy equation. J. Differ. Equ. 124(1), 225-246 (1996)

[10] Cléry, F., Ferapontov, E.V.: Dispersionless Hirota equations and the genus 3 hyperelliptic divisor. Commun. Math. Phys. 376(2), 1397-1412 (2020)

[11] Donato, A., Ramgulam, U., Rogers, C.: The $(3+1)$-dimensional Monge-Ampère equation in discontinuity wave theory: application of a reciprocal transformation. Meccanica 27, 257-262 (1992)

[12] Dunajski, M., Ferapontov, E.V., Kruglikov, B.: On the Einstein-Weyl and conformal self-duality equations. J. Math. Phys. 56, 083501 (2015)

[13] Dunajski, M., Tod, P.: Einstein-Weyl spaces and dispersionless KadomtsevPetviashvili equation from Painlevé I and II. Phys. Lett. A 303, 253-264 (2002)

[14] Dunajski, M.: An interpolating dispersionless integrable system. J. Phys. A 4(31), 315202 (2008)

[15] Dunajski, M., Mason, L.J., Tod, P.: Einstein-Weyl geometry, the dKP equation and twistor theory. J. Geom. Phys. 37(1-2), 63-93 (2001)

[16] Dunajski, M., Tod, P.: Einstein-Weyl structures from hyper-Kähler metrics with conformal Killing vectors. Differ. Geom. Appl. 14(1), 39-55 (2001)

[17] Dunajski, M.: A class of Einstein-Weyl spaces associated to an integrable system of hydrodynamic type. J. Geom. Phys. 51(1), 126-137 (2004)

[18] Eastwood, M.G., Tod, K.P.: Local constraints on Einstein-Weyl geometries. J. Reine Angew. Math. 491, 183-198 (1997)

[19] Eastwood, M.G., Tod, K.P.: Local constraints on Einstein-Weyl geometries: the 3-dimensional case. Ann. Global Anal. Geom. 18(1), 1-27 (2000)

[20] Ferapontov, E.V., Habibullin, I.T., Kuznetsova, M.N., Novikov, V.S.: On a class of 2D integrable lattice equations. J. Math. Phys. 61(7), 073505 (2020)

[21] Ferapontov, E.V., Kruglikov, B.: Dispersionless integrable systems in 3D and Einstein-Weyl geometry. J. Differ. Geom. 97, 215-254 (2014)

[22] Ferapontov, E.V., Khusnutdinova, K.R.: On the integrability of (2+1)dimensional quasilinear systems. Commun. Math. Phys. 248, 187-206 (2004)

[23] Ferapontov, E.V., Kruglikov, B., Novikov, V.S.: Integrability of dispersionless Hirota type equations in $4 \mathrm{D}$ and the symplectic Monge-Ampère property. Int. Math. Res. Notices (2021). https://doi.org/10.1093/imrn/rnaa025

[24] Ferapontov, E.V., Hadjikos, L., Khusnutdinova, K.R.: Integrable equations of the dispersionless Hirota type and hypersurfaces in the Lagrangian Grassmannian. Int. Math. Res. Notices 2010(3), 496-535 (2010) 
[25] Ferapontov, E.V., Odesskii, A.V.: Integrable Lagrangians and modular forms. J. Geom. Phys. 60(6-8), 896-906 (2010)

[26] Gutt, J., Manno, G., Moreno, G.: Completely exceptional 2nd order PDEs via conformal geometry and BGG resolution. J. Geom. Phys. 113, 86-103 (2017)

[27] Hitchin, N.J.: Complex manifolds and Einstein's equations, Twistor geometry and nonlinear systems (Primorsko, 1980), 73-99. Lecture Notes in Mathematics, vol. 970. Springer, Berlin (1982)

[28] Jones, P.E., Tod, K.P.: Minitwistor spaces and Einstein-Weyl spaces. Class. Quant. Gr. 2(4), 565-577 (1985)

[29] Kruglikov, B., Panasyuk, A.: Veronese webs and nonlinear PDEs. J. Geom. Phys. 115, 45-60 (2017)

[30] Kryński, W.: On deformations of the dispersionless Hirota equation. J. Geom. Phys. 127, 46-54 (2018)

[31] Pavlov, M.V.: New integrable (2+1)-equations of hydrodynamic type. Russ. Math. Surv. 58(2), 384-385 (2003)

[32] Ruggeri, T.: Su una naturale estensione a tre variabili dell' equazione di MongeAmpère, pp. 445-449. LV, Accad. Naz. Lincei (1973)

[33] Ward, R.S.: Einstein-Weyl spaces and $S U(\infty)$ Toda fields. Class. Quant. Grav. 7(4), L95-L98 (1990)

[34] Zakharevich, I.: Nonlinear wave equation, nonlinear Riemann problem, and the twistor transform of Veronese webs (2000). arXiv:math-ph/0006001

[35] Zakharov, V.E.: Dispersionless limit of integrable systems in $2+1$ dimensions. In: Ercolani, N.M., et al. (eds.) Singular Limits of Dispersive Waves, pp. 165-174. Plenum Press, New York (1994)

S. Berjawi, E. V. Ferapontov and V. S. Novikov

Department of Mathematical Sciences

Loughborough University

Loughborough

Leicestershire

LE11 3TU

UK

e-mail: S.Berjawi@lboro.ac.uk;

V.Novikov@lboro.ac.uk

E. V. Ferapontov

Institute of Mathematics, Ufa Federal Research Centre

Russian Academy of Sciences

112 Chernyshevsky Street

Ufa

Russian Federation 450008

e-mail: E.V.Ferapontov@lboro.ac.uk 
B. S. Kruglikov

Department of Mathematics and Statistics, Faculty of Natural Sciences UiT the Arctic University of Norway

90-37 Tromsö

Norway

e-mail: Boris.Kruglikov@uit.no

and

Department of Mathematics and Natural Sciences

University of Stavanger

40-36 Stavanger

Norway

Communicated by Nikolai Kitanine.

Received: June 16, 2021.

Accepted: November 18, 2021. 